\title{
The boundary motive: definition and basic properties
}

\author{
Jörg Wildeshaus
}

\begin{abstract}
We introduce the notion of the boundary motive of a scheme $X$ over a perfect field. By definition, it measures the difference between the motive $M_{\mathrm{gm}}(X)$ and the motive with compact support $M_{\mathrm{gm}}^{\mathrm{c}}(X)$, as defined and studied by Voevodsky et al. in Cycles, transfers, and motivic homology theories (Annals of Mathematics Studies, vol. 143 (Princeton University Press, Princeton, NJ, 2000)). We develop three tools to compute the boundary motive in terms of the geometry of a compactification of $X$ : co-localization; invariance under abstract blow-up; and analytical invariance. We then prove auto-duality of the boundary motive of a smooth scheme $X$. As a formal consequence of this, and of co-localization, we obtain a fourth computational tool, namely localization for the boundary motive. In a sequel to this work (J. Wildeshaus, On the boundary motive of a Shimura variety, Prépublications du Laboratoire d'Analyse, Géométrie et Applications de l'Université Paris 13, no. 2004-23), these tools will be applied to Shimura varieties.
\end{abstract}

\section{Contents}

Introduction

631

1 Notation and conventions $\quad 633$

2 Definition of the boundary motive $\quad 635$

3 Co-localization $\quad 636$

4 Invariance under abstract blow-up $\quad 637$

$\begin{array}{lll}5 & \text { Analytical invariance } & 638\end{array}$

6 Auto-duality $\quad 641$

7 Localization $\quad 652$

8 The case of normal crossings $\quad 653$

$\begin{array}{ll}\text { References } & 656\end{array}$

\section{Introduction}

In this paper, we define the boundary motive $\partial M_{\mathrm{gm}}(X)$ of a scheme $X$ over a perfect field $k$. By its very construction, it is part of an exact triangle

$$
\partial M_{\mathrm{gm}}(X) \longrightarrow M_{\mathrm{gm}}(X) \longrightarrow M_{\mathrm{gm}}^{\mathrm{c}}(X) \longrightarrow \partial M_{\mathrm{gm}}(X)[1],
$$

where $M_{\mathrm{gm}}(X)$ and $M_{\mathrm{gm}}^{\mathrm{c}}(X)$ denote the motive of $X$ and its motive with compact support, respectively. The exact triangle is in the triangulated category $D M_{-}^{\text {eff }}(k)$ of effective motivic complexes which, together with $M_{\mathrm{gm}}(X)$ and $M_{\mathrm{gm}}^{\mathrm{c}}(X)$, was defined in [VSF00]. We refer to $\S 1$ for a review

Received 2 April 2005, accepted in final form 15 August 2005.

2000 Mathematics Subject Classification 14F42 (primary), 14C25, $19 \mathrm{E} 15$ (secondary).

Keywords: finite correspondences, Nisnevich sheaves with transfers, motives, motives with compact support.

This journal is (C) Foundation Compositio Mathematica 2006. 


\section{J. Wildeshaus}

of these constructions. We expect this exact triangle to be of a certain interest. First, it induces long exact sequences for motivic homology and cohomology. More generally, any exact functor to a triangulated category $D$ will induce long exact sequences of Ext-groups in $D$ which are a priori compatible with the sequence in motivic homology, respectively, cohomology. Second, the exact triangle itself can be employed to construct explicit extensions of objects in $D M_{-}^{\text {eff }}(k)$, i.e. classes in motivic cohomology.

Note that most of the existing attempts to prove the Beilinson or Bloch-Kato conjectures on special values of $L$-functions necessitate the explicit construction of elements in motivic cohomology. Furthermore, the realizations (Betti, de Rham, étale,... ) of these elements can often be constructed out from the cohomology of non-compact varieties, using the respective realizations of our exact triangle

$$
\partial M_{\mathrm{gm}}(X) \longrightarrow M_{\mathrm{gm}}(X) \longrightarrow M_{\mathrm{gm}}^{\mathrm{c}}(X) \longrightarrow \partial M_{\mathrm{gm}}(X)[1] .
$$

This approach is clearly present, for example, in Harder's work on special values [Har93]. Our definition may thus be seen as an attempt to give a rigorous motivic meaning to these constructions.

In order to efficiently apply our new notion, one is naturally led to look for means to identify the boundary motive. We develop three tools to compute $\partial M_{\mathrm{gm}}(X)$ : co-localization $(\S 3)$; invariance under abstract blow-up $(\S 4)$; and analytical invariance $(\S 5)$. In a sequel to this work [Wil04], they will be applied to Shimura varieties, yielding in particular a motivic version of Pink's theorem on higher direct images of étale sheaves in the Baily-Borel compactification.

All our tools are based on the identification of $\partial M_{\mathrm{gm}}(X)$ with the motive associated to the diagram of schemes

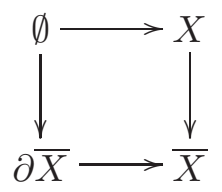

for a compactification $\bar{X}$ of $X$, with $\partial \bar{X}:=\bar{X}-X$ (see Proposition 2.4 for the precise statement). Let us insist that the definition of $\partial M_{\mathrm{gm}}(X)$ does not involve such a compactification.

Co-localization is the motivic analogue of the dual of the localization spectral sequence associated to a stratification of $\partial \bar{X}$. In the context of Betti cohomology (say), a good stratification $\partial \bar{X}=\coprod Y_{\mathrm{m}}$ induces a spectral sequence converging to the cohomology of $\partial \bar{X}$, and with $E_{2}$-terms given by cohomology with compact support of the $Y_{\mathrm{m}}$. If one wants to express the analogue of the $E_{2}$-terms as motives rather than motives with compact support, one is led to imitate the dual spectral sequence, whose $E_{2}$-terms are equal to the usual cohomology of $Y_{\mathrm{m}}$, but with coefficients given by the exceptional inverse images of the coefficients on $\partial \bar{X}$. This explains our choice of notation for the motives 'with coefficients' occurring in $\S \S 3-5$, and more importantly, the precise nature of their behaviour. It turns out that in order to obtain a clean statement, co-localization (Theorem 3.4) has to be formulated more generally for diagrams of the type

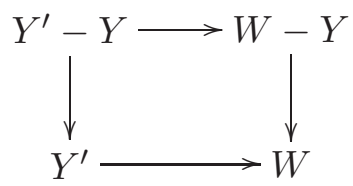

and for stratifications of $Y$, where $Y \hookrightarrow Y^{\prime} \hookrightarrow W$ are closed immersions of not necessarily proper schemes over $k$. This diagram should be seen as modeling cohomology of the 'motivic sheaf' $i_{Y}^{!} j ! \mathbb{Z}$, where $i_{Y}: Y \hookrightarrow W$ and $j: W-Y^{\prime} \hookrightarrow W$. Due to the lack of sufficient covariance properties of the functor $M_{\mathrm{gm}}^{\mathrm{c}}$, it does not seem obvious to geometrically model cohomology with compact support of $i_{Y}^{*} j_{*} \mathbb{Z}$. We refer to Definition 7.1 for a partial remedy to this problem. 


\section{THE BOUNDARY MOTIVE: DEFINITION AND BASIC PROPERTIES}

Given the sheaf-theoretical point of view, invariance under abstract blow-up and analytical invariance come as no surprise: in the context of complex spaces (say), the cohomology of $i_{Y}^{!} j_{!} \mathbb{Z}$ can be computed after proper base change to a second diagram as above, provided that this base change induces an isomorphism above the complement $W-Y^{\prime}$. Theorem 4.1 is the motivic analogue of this invariance. Verdier's theory of the specialization functor (see e.g. the overview in [Ver85, $\S 1]$ ) shows that the sheaf $i_{Y}^{!} j_{!} \mathbb{Z}$ on $Y$ can be computed on the normal cone of $Y$ along $W$. Since analytically isomorphic situations near $Y$ lead to the same normal cone, this implies that $i_{Y}^{!} j_{!} \mathbb{Z}$ can be computed with respect to a second set of closed immersions $Y \hookrightarrow Y_{2}^{\prime} \hookrightarrow W_{2}$, provided that the formal completions of $W_{2}$, respectively of $Y_{2}^{\prime}$, along $Y$ agree with those of $W$, respectively of $Y^{\prime}$, along $Y$. Theorem 5.1 states that the motivic analogue of this latter statement holds. While co-localization and invariance under abstract blow-up are direct consequences of the material contained in [VSF00], the proof of analytical invariance in addition uses the full force of Artin approximation.

In $\S 6$, we generalize duality for bivariant cycle cohomology [VSF00, Theorem IV.7.4], in order to establish an important structural property of the boundary motive $\partial M_{\mathrm{gm}}(X)$ of a smooth scheme $X$ of pure dimension $n$ : it is canonically isomorphic to its own dual $\partial M_{\mathrm{gm}}(X)^{*}$, twisted by $n$ and shifted by $2 n-1$ (Theorem 6.1). As one formal consequence of this auto-duality, and of co-localization, we obtain a fourth tool to compute $\partial M_{\mathrm{gm}}(X)$, namely localization $(\S 7)$ in the context of stratifications $\partial \bar{X}=\amalg Y_{\mathrm{m}}$, for a compactification $\bar{X}$ of a smooth scheme $X$ (with $\partial \bar{X}=\bar{X}-X$ as above).

Sections 3-6 are logically independent of each other. Section 1 serves as a basis for all that is to follow, and $\S 7$ uses everything said before. All results from $\S \S 4,6$ and 7 require resolution of singularities for the base field $k$.

\section{Notation and conventions}

Our main and almost only reference is the book [VSF00]. When citing a result from its Chapter $n$, we shall precede the numbering used in [VSF00] by $N$, where $N$ is the symbol representing $n$ in the Roman number system. For example: Proposition 3.1.3 from Chapter 5 from [VSF00] will be cited as [VSF00, Proposition V.3.1.3].

We follow the notation of [VSF00]. Fix a perfect base field $k$. Denote by $S c h / k$ the category of schemes which are separated and of finite type over $k$, and by $S m / k$ the full sub-category of objects which are smooth over $k$. Recall the definition of the category $\operatorname{Sm} \operatorname{Cor}(k)$ [VSF00, p. 190]: its objects are those of $S m / k$. Morphisms from $Y$ to $X$ are given by the group $c(Y, X)$ of finite correspondences from $Y$ to $X$, defined as the free Abelian group on the symbols $(Z)$, where $Z$ runs through the integral closed sub-schemes of $Y \times_{k} X$ which are finite over $Y$ and surjective over a connected component of $Y$. Note for later use that the definition of $c(Y, X)$ still makes sense when $X \in S c h / k$ is not necessarily smooth. The category $\operatorname{Sh} v_{\text {Nis }}(\operatorname{Sm} \operatorname{Cor}(k))$ of Nisnevich sheaves with transfers [VSF00, Definition V.3.1.1] is the category of those contravariant additive functors from $\operatorname{SmCor}(k)$ to Abelian groups, whose restriction to $S m / k$ is a sheaf for the Nisnevich topology. This category is Abelian [VSF00, Theorem V.3.1.4]. Inside the derived category $D^{-}\left(\operatorname{Sh} v_{\text {Nis }}(\operatorname{SmCor}(k))\right)$ of complexes bounded from above, one defines the full sub-category $D M_{-}^{\text {eff }}(k)$ of effective motivic complexes over $k$ [VSF00, p. 205] as the one consisting of objects whose cohomology sheaves are homotopy invariant [VSF00, Definition V.3.1.10]. Since $k$ is supposed to be perfect, this sub-category is triangulated [VSF00, Proposition V.3.1.13]. According to [VSF00, Proposition V.3.2.3], the inclusion of $D M_{-}^{\text {eff }}(k)$ into $D^{-}\left(S h v_{\text {Nis }}(S m C o r(k))\right)$ admits a left adjoint $\mathbf{R} C$, which is induced from the functor

$$
\underline{C}_{*}: S h v_{\mathrm{Nis}}(\operatorname{SmCor}(k)) \longrightarrow C^{-}\left(\operatorname{Sh} v_{\mathrm{Nis}}(\operatorname{Sm} \operatorname{Cor}(k))\right)
$$

which maps $F$ to the simple complex associated to the singular simplicial complex [VSF00, p. 207]. Its $n$th term (in homological numbering) $\underline{C}_{n}(F)$ sends $X$ to $F\left(X \times_{k} \Delta^{n}\right)$. 


\section{J. Wildeshaus}

One defines two functors $L$ and $L^{\mathrm{c}}$ from $S c h / k$ to $S h v_{\mathrm{Nis}}(\operatorname{Sm} \operatorname{Cor}(k))$ [VSF00, pp. 223-224]: the functor $L$ associates to $X$ the Nisnevich sheaf with transfers $c(\bullet, X)$. The functor $L^{\mathrm{c}}$ maps $X$ to

$$
Y \longmapsto z(Y, X),
$$

$z(Y, X)$ being defined as the free Abelian group on the symbols $(Z)$, where $Z$ runs through the integral closed sub-schemes of $Y \times_{k} X$ which are quasi-finite over $Y$ and dominant over a connected component of $Y$. One defines the motive $M_{\mathrm{gm}}(X)$ of $X \in S c h / k$ as $\mathbf{R} C(L(X))$, and the motive with compact support $M_{\mathrm{gm}}^{\mathrm{c}}(X)$ as $\mathbf{R} C\left(L^{\mathrm{c}}(X)\right)$.

For certain applications, it is of interest to enlarge the domain of the functor $L$ : denote by $S c h^{\infty} / k$ the category of schemes which are separated and locally of finite type over $k$. The functor $L$ extends, with the same definition of $c(\bullet, X)$ as above. This identifies $L(X)$ with the filtered direct limit of the $L(U)$, with $U$ running through the open sub-schemes of $X$ which are of finite type over $k$. This observation also allows us to use certain results from [VSF00] for the motives $M_{\mathrm{gm}}(X):=\mathbf{R} C(L(X)) \in D M_{-}^{\mathrm{eff}}(k)$, with $X$ in $S c h^{\infty} / k$.

We shall also use another, more geometric approach to motives, i.e. the one developed in [VSF00, $\S$ V.2.1]. There, the triangulated category $D M_{\mathrm{gm}}^{\mathrm{eff}}(k)$ of effective geometrical motives over $k$ is defined. There is a canonical full triangulated embedding of $D M_{\mathrm{gm}}^{\mathrm{eff}}(k)$ into $D M_{-}^{\mathrm{eff}}(k)$ [VSF00, Theorem V.3.2.6], which maps the geometrical motive of $X \in S m / k$ [VSF00, Definition V.2.1.1] to $M_{\mathrm{gm}}(X)$. Using this embedding, we consider $M_{\mathrm{gm}}(X)$ as an object of $D M_{\mathrm{gm}}^{\mathrm{eff}}(k)$. Finally, the category $D M_{\mathrm{gm}}(k)$ of geometrical motives over $k$ is obtained from $D M_{\mathrm{gm}}^{\mathrm{eff}}(k)$ by inverting the Tate motive $\mathbb{Z}(1)$ [VSF00, p. 192]. All four categories $D M_{\mathrm{gm}}^{\mathrm{eff}}(k), D M_{\mathrm{gm}}(k), D^{-}\left(\operatorname{Sh} v_{\mathrm{Nis}}(\operatorname{SmCor}(k))\right)$, and $D M_{-}^{\mathrm{eff}}(k)$ are tensor triangulated, and admit unit objects [VSF00, Proposition V.2.1.3, Corollary V.2.1.5, p. 206, Theorem V.3.2.6]. These tensor structures are such that for all $X, Y \in S m / k$, one has

$$
M_{\mathrm{gm}}(X) \otimes M_{\mathrm{gm}}(Y)=M_{\mathrm{gm}}\left(X \times_{k} Y\right)
$$

in $D M_{\mathrm{gm}}^{\mathrm{eff}}(k)$, and

$$
L(X) \otimes L(Y)=L\left(X \times_{k} Y\right)
$$

in $D^{-}\left(S h v_{\mathrm{Nis}}(\operatorname{SmCor}(k))\right)$. The unit object of $D M_{\mathrm{gm}}^{\mathrm{eff}}(k)$ is $M_{\mathrm{gm}}(\operatorname{Spec} k)$, and that of $D^{-}\left(S h v_{\mathrm{Nis}}\right.$ $(\operatorname{SmCor}(k)))$ is $L(\operatorname{Spec} k)$. Both of them are denoted by $\mathbb{Z}(0)$. For $M \in D M_{\mathrm{gm}}(k)$ and $n \in \mathbb{Z}$, write $M(n)$ for the tensor product $M \otimes \mathbb{Z}(n)$. The three functors $D M_{\mathrm{gm}}^{\mathrm{eff}}(k) \hookrightarrow D M_{-}^{\mathrm{eff}}(k), D M_{\mathrm{gm}}^{\mathrm{eff}}(k) \rightarrow$ $D M_{\mathrm{gm}}(k)$ and $\mathbf{R} C: D^{-}\left(S h v_{\mathrm{Nis}}(\operatorname{SmCor}(k))\right) \rightarrow D M_{-}^{\text {eff }}(k)$ are compatible with the tensor structure. (By contrast, the embedding of $D M_{-}^{\text {eff }}(k)$ into $D^{-}\left(\operatorname{Sh} v_{\text {Nis }}(\operatorname{SmCor}(k))\right)$ is not; see [VSF00, Remark on p. 206].) According to [VSF00, Theorem V.4.3.1], the functor $D M_{\mathrm{gm}}^{\mathrm{eff}}(k) \rightarrow D M_{\mathrm{gm}}(k)$ is a full triangulated embedding if $k$ admits resolution of singularities.

CONVEntion 1.1. Whenever we speak about resolution of singularities, it will be taken in the sense of [VSF00, Definition IV.3.4].

Convention 1.2. We shall use the same symbol for $M_{\mathrm{gm}}(X) \in D M_{-}^{\text {eff }}(k)$ and for its canonical representative $\underline{C}_{*}(L(X))$ in $C^{-}\left(S h v_{\mathrm{Nis}}(S m \operatorname{Cor}(k))\right)$; similarly for $M_{\mathrm{gm}}^{\mathrm{c}}(X)$. Whenever we speak about cones of morphisms between motives, we mean the class of the cone of the morphism between the canonical representatives. For a commutative diagram

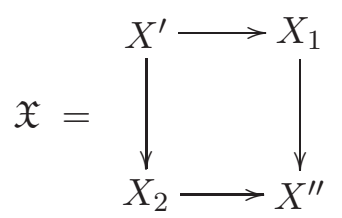

in $S c h^{\infty} / k$, define its motive $M_{\mathrm{gm}}(\mathfrak{X}) \in D M_{-}^{\mathrm{eff}}(k)$ as $\mathbf{R} C$ applied to the simple complex $s L(\mathfrak{X})$ 


\section{THE BOUNDARY MOTIVE: DEFINITION AND BASIC PROPERTIES}

associated to $L(\mathfrak{X})$, i.e. to the complex

$$
L\left(X^{\prime}\right) \longrightarrow L\left(X_{1}\right) \oplus L\left(X_{2}\right) \longrightarrow L\left(X^{\prime \prime}\right),
$$

which we normalize by assigning degree zero to the component $L\left(X^{\prime \prime}\right)$. A similar construction is possible for commutative diagrams in $S c h^{\infty} / k$ of 'dimension' greater than two, provided that there are not more than two schemes on any of the lines in the diagram.

\section{Definition of the boundary motive}

Let $X \in S c h / k$. Note that the inclusion $c(\bullet, X) \hookrightarrow z(\bullet, X)$ induces a monomorphism

$$
\iota_{X}: L(X) \longleftrightarrow L^{\mathrm{c}}(X)
$$

Definition 2.1. The boundary motive of $X$ is defined as

$$
\partial M_{\mathrm{gm}}(X):=\mathbf{R} C\left(\operatorname{Coker} \iota_{X}\right)[-1] .
$$

Note that there is a canonical quasi-isomorphism

$$
\operatorname{Cone}\left(M_{\mathrm{gm}}(X) \longrightarrow M_{\mathrm{gm}}^{\mathrm{c}}(X)\right) \longrightarrow \partial M_{\mathrm{gm}}(X)[1],
$$

where the cone is to be understood as in Convention 1.2. We have the following.

Proposition 2.2. There is an exact triangle

$$
\partial M_{\mathrm{gm}}(X) \longrightarrow M_{\mathrm{gm}}(X) \longrightarrow M_{\mathrm{gm}}^{\mathrm{c}}(X) \longrightarrow \partial M_{\mathrm{gm}}(X)[1]
$$

in $D M_{-}^{\text {eff }}(k)$.

Corollary 2.3. Assume that $k$ admits resolution of singularities. Then the boundary motive $\partial M_{\mathrm{gm}}(X)$ belongs to $D M_{\mathrm{gm}}^{\mathrm{eff}}(k)$.

Proof. This follows from Proposition 2.2, the fact that the embedding of $D M_{\mathrm{gm}}^{\mathrm{eff}}(k)$ into $D M_{-}^{\mathrm{eff}}(k)$ is triangulated, and [VSF00, Corollary V.4.1.4 and Corollary V.4.1.6].

The definition of the boundary motive does not involve a compactification of $X$. However, the tools to compute $\partial M_{\mathrm{gm}}(X)$ which we shall develop in the sequel are based on the following.

Proposition 2.4. Let $\bar{X}$ be a compactification of $X \in S c h / k$, and define $\partial \bar{X}$ as the complement $\bar{X}-X$, equipped with the reduced scheme structure. There is a canonical morphism

$$
\text { Cone }\left(M_{\mathrm{gm}}(X \coprod \partial \bar{X}) \rightarrow M_{\mathrm{gm}}(\bar{X})\right) \longrightarrow \partial M_{\mathrm{gm}}(X)[1]
$$

in $C^{-}\left(S h v_{\text {Nis }}(\operatorname{SmCor}(k))\right)$. It becomes an isomorphism in $D M_{-}^{\text {eff }}(k)$ if $k$ admits resolution of singularities.

Proof. Consider the exact sequences

$$
0 \longrightarrow L(X) \longrightarrow L^{\mathrm{c}}(X) \longrightarrow \text { Coker } \iota_{X} \longrightarrow 0
$$

and

$$
0 \longrightarrow L^{\mathrm{c}}(\partial \bar{X}) \longrightarrow L^{\mathrm{c}}(\bar{X}) \longrightarrow L^{\mathrm{c}}(X)
$$

Observe that since $\partial \bar{X}$ and $\bar{X}$ are proper, we have $L(\partial \bar{X})=L^{\mathrm{c}}(\partial \bar{X})$ and $L(\bar{X})=L^{\mathrm{c}}(\bar{X})$. The monomorphism $L(X) \hookrightarrow L^{\mathrm{c}}(X)$ factors through $L(\bar{X})$. Hence the exact sequences induce a monomorphism between the quotient $L(\bar{X}) / L(X \coprod \partial \bar{X})$ and Coker $\iota_{X}$, whose cokernel is identical to that of the restriction $L(\bar{X})=L^{\mathrm{c}}(\bar{X}) \rightarrow L^{\mathrm{c}}(X)$. According to [VSF00, Proposition V.4.1.5], this latter cokernel has trivial image under $\mathbf{R} C$ if $k$ admits resolution of singularities. 


\section{J. WiLDESHAUS}

\section{Co-localization}

Consider the geometric situation of Proposition 2.4: let $X \in S c h / k$, choose a compactification $\bar{X}$ of $X$, and define $\partial \bar{X}:=\bar{X}-X$. In this section, we develop the motivic analogue of the dual of the localization spectral sequence associated to a stratification of $\partial \bar{X}$. It turns out to be useful to consider a more general geometric situation: fix closed immersions $Y \hookrightarrow Y^{\prime} \hookrightarrow W$ in $S c h^{\infty} / k$. Write $j$ for the open immersion of $W-Y^{\prime}$, and $i_{Y}$ for the closed immersion of $Y$ into $W$. Denote the following commutative diagram by $\mathfrak{Y}$.

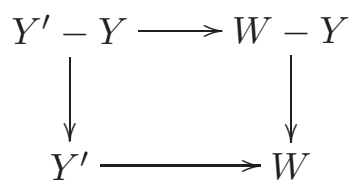

Definition 3.1. The motive of $Y$ with coefficients in $i_{Y}^{!} j ! \mathbb{Z}$ is defined as

$$
M_{\mathrm{gm}}\left(Y, i_{Y}^{!} j_{!} \mathbb{Z}\right):=M_{\mathrm{gm}}(\mathfrak{Y})
$$

(see Convention 1.2).

Remark 3.2.

(a) Note that $M_{\mathrm{gm}}\left(Y, i_{Y}^{!} j_{!} \mathbb{Z}\right)$ lies in $D M_{\mathrm{gm}}^{\mathrm{eff}}(k)$ if $W$ and $Y^{\prime}$ are in $S m / k$.

(b) Assume that $k$ admits resolution of singularities. Then $M_{\mathrm{gm}}\left(Y, i_{Y}^{!} j ! \mathbb{Z}\right)$ lies in $D M_{\mathrm{gm}}^{\mathrm{eff}}(k)$ if $W$ is in $S c h / k$ [VSF00, Corollary V.4.1.4].

Remark 3.3. If $Y=Y^{\prime}$, i.e. if $i_{Y}$ is complementary to $j$, then $\mathfrak{Y}$ acquires the form

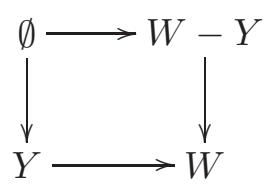

and we have

$$
M_{\mathrm{gm}}\left(Y, i_{Y}^{!} j_{!} \mathbb{Z}\right)=\text { Cone }\left(M_{\mathrm{gm}}((W-Y) \coprod Y) \rightarrow M_{\mathrm{gm}}(W)\right) .
$$

If in addition $W$ is proper, then Proposition 2.4 relates $M_{\mathrm{gm}}\left(Y, i_{Y}^{!} j_{!} \mathbb{Z}\right)$ to $\partial M_{\mathrm{gm}}(W-Y)[1]$.

Now assume a given filtration

$$
\emptyset=\mathfrak{F}_{-1} Y \subset \mathfrak{F}_{0} Y \subset \cdots \subset \mathfrak{F}_{d} Y=Y
$$

of $Y$ by closed sub-schemes. It induces a stratification of $Y$ by locally closed sub-schemes $Y_{\mathrm{m}}:=$ $\mathfrak{F}_{\mathrm{m}} Y-\mathfrak{F}_{\mathrm{m}-1} Y$, for $m=0, \ldots, d$. Define $W^{m}$ as the complement of $\mathfrak{F}_{\mathrm{m}-1} Y$ in $W$. This gives a descending partial filtration of $W$ by open sub-schemes. Note in particular that we have $W^{0}=W$ and $W^{d+1}=W-Y$. Write $i_{Y_{\mathrm{m}}}$ for the closed immersion of $Y_{\mathrm{m}}$ into $W^{m}$. By abuse of notation, we use the letter $j$ to also denote the open immersions of $W-Y^{\prime}$ into $W^{m}$.

Theorem 3.4 (Co-localization). There is a canonical chain of morphisms

$$
M^{d+1}=0 \stackrel{\gamma^{d}}{\longrightarrow} M^{d} \stackrel{\gamma^{d-1}}{\longrightarrow} M^{d-1} \stackrel{\gamma^{d-2}}{\longrightarrow} \cdots \stackrel{\gamma^{0}}{\longrightarrow} M^{0}=M_{\mathrm{gm}}\left(Y, i_{Y}^{!} j ! \mathbb{Z}\right)
$$

in $C^{-}\left(S h v_{\mathrm{Nis}}(\operatorname{SmCor}(k))\right)$. For each $m \in\{0, \ldots, d\}$, there is a canonical isomorphism

$$
\text { Cone } \gamma^{m} \cong M_{\mathrm{gm}}\left(Y_{\mathrm{m}}, i_{Y_{\mathrm{m}}} j ! \mathbb{Z}\right)
$$

and hence a canonical exact triangle

$$
M_{\mathrm{gm}}\left(Y_{\mathrm{m}}, i_{Y_{\mathrm{m}}}^{!} j_{!} \mathbb{Z}\right)[-1] \longrightarrow M^{m+1} \stackrel{\gamma^{m}}{\longrightarrow} M^{m} \longrightarrow M_{\mathrm{gm}}\left(Y_{\mathrm{m}}, i_{Y_{\mathrm{m}}}^{!} j_{!} \mathbb{Z}\right)
$$




\section{THE BOUNDARY MOTIVE: DEFINITION AND BASIC PROPERTIES}

in $D M_{-}^{\text {eff }}(k)$. In particular, all the $M^{m}$ represent objects in $D M_{-}^{\text {eff }}(k)$. If $k$ admits resolution of singularities, then all the $M^{m}$ represent objects in $D M_{\mathrm{gm}}^{\mathrm{eff}}(k)$.

Proof. Consider the induced filtration $Y^{\prime m}:=Y^{\prime}-\mathfrak{F}_{\mathrm{m}-1} Y=W^{m} \cap Y^{\prime}$, and define $M^{m}:=M_{\mathrm{gm}}\left(\mathfrak{Y}^{m}\right)$, where $\mathfrak{Y}^{m}$ is the following commutative diagram.

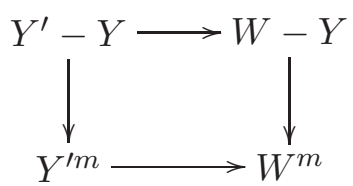

Now note that $M_{\mathrm{gm}}\left(Y_{\mathrm{m}}, i_{Y_{\mathrm{m}}}^{!} j_{!} \mathbb{Z}\right)$ is the motive associated to the following diagram.

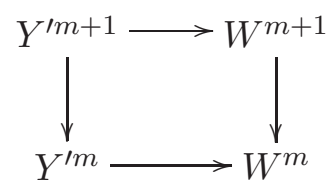

Corollary 3.5. Assume that $k$ admits resolution of singularities. In the above situation, assume that $Y=\partial \bar{X}:=\bar{X}-X$, with $X \in S c h / k$, and $\bar{X}$ a compactification of $X$. Write $(\partial \bar{X})_{\mathrm{m}}:=Y_{\mathrm{m}}$. Then there is a canonical chain of morphisms

$$
M^{d+1}=0 \stackrel{\gamma^{d}}{\longrightarrow} M^{d} \stackrel{\gamma^{d-1}}{\longrightarrow} M^{d-1} \stackrel{\gamma^{d-2}}{\longrightarrow} \cdots \stackrel{\gamma^{0}}{\longrightarrow} M^{0}=\partial M_{\mathrm{gm}}(X)[1]
$$

in $C^{-}\left(S h v_{\text {Nis }}(\operatorname{SmCor}(k))\right)$. For each $m \in\{0, \ldots, d\}$, there is a canonical exact triangle

$$
M_{\mathrm{gm}}\left((\partial \bar{X})_{\mathrm{m}}, i_{(\partial \bar{X})_{\mathrm{m}}}^{!} j_{!} \mathbb{Z}\right)[-1] \rightarrow M^{m+1} \stackrel{\gamma^{m}}{\longrightarrow} M^{m} \rightarrow M_{\mathrm{gm}}\left((\partial \bar{X})_{\mathrm{m}}, i_{(\partial \bar{X})_{\mathrm{m}}}^{!} j_{!} \mathbb{Z}\right)
$$

in $D M_{\mathrm{gm}}(k)$. In particular, all the $M^{m}$ are in $D M_{\mathrm{gm}}^{\mathrm{eff}}(k)$.

Proof. This follows from Proposition 2.4 and Theorem 3.4.

Of particular interest is the case when $X \in S m / k$, and $\bar{X}$ is a smooth compactification of $X$, such that $\partial \bar{X}$ is a normal crossing divisor. Then $d<\operatorname{dim} X$ and $(\partial \bar{X})_{\mathrm{m}}$ equals the geometric locus of points lying on exactly $d+1-m$ local irreducible components of $\partial \bar{X}$. Since analytical invariance will permit us to give a good description of the $M_{\mathrm{gm}}\left((\partial \bar{X})_{\mathrm{m}}, i_{(\partial \bar{X})_{\mathrm{m}}}^{!} j_{!} \mathbb{Z}\right)$, we postpone the discussion of this case until $\S 8$.

\section{Invariance under abstract blow-up}

Fix a proper morphism $\pi: W_{1} \rightarrow W_{2}$ and closed immersions $Y_{2} \hookrightarrow Y_{2}^{\prime} \hookrightarrow W_{2}$ in $S c h^{\infty} / k$. Write $Y_{1} \hookrightarrow$ $Y_{1}^{\prime} \hookrightarrow W_{1}$ for the base change of these immersions, $j_{\mathrm{m}}$ for the open immersion of $W_{\mathrm{m}}-Y_{\mathrm{m}}^{\prime}$ and $i_{Y_{\mathrm{m}}}$ for the closed immersion of $Y_{\mathrm{m}}$ into $W_{\mathrm{m}}$. We obtain the following diagram.

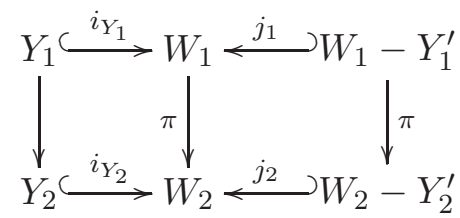

Theorem 4.1 (Invariance under abstract blow-up). Assume that

$$
\pi: W_{1}-Y_{1}^{\prime} \longrightarrow W_{2}-Y_{2}^{\prime}
$$

is an isomorphism. If $k$ admits resolution of singularities, then the map

$$
\pi: M_{\mathrm{gm}}\left(Y_{1}, i_{Y_{1}}^{!} j_{1 !} \mathbb{Z}\right) \longrightarrow M_{\mathrm{gm}}\left(Y_{2}, i_{Y_{2}}^{!} j_{2 !} \mathbb{Z}\right)
$$

is an isomorphism. 


\section{J. WiLdeshaus}

Recall that $M_{\mathrm{gm}}\left(Y_{\mathrm{m}}, i_{Y_{\mathrm{m}}}^{!} j_{\mathrm{m} !} \mathbb{Z}\right)$ is associated to the following diagram.

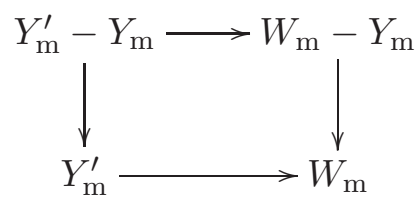

Since the two rows are of the same nature, Theorem 4.1 is a formal consequence of the following.

Theorem 4.2. Consider closed immersions $Y_{1}^{\prime} \hookrightarrow W_{1}$ and $Y_{2}^{\prime} \hookrightarrow W_{2}$ in $S c h^{\infty} / k$, and a proper morphism $\pi: W_{1} \rightarrow W_{2}$ identifying $Y_{1}^{\prime}$ with the fibre product $W_{1} \times_{W_{2}} Y_{2}^{\prime}$, and inducing an isomorphism from $W_{1}-Y_{1}^{\prime}$ to $W_{2}-Y_{2}^{\prime}$. If $k$ admits resolution of singularities, then the monomorphism

$$
\pi: L\left(W_{1}\right) / L\left(Y_{1}^{\prime}\right) \longrightarrow L\left(W_{2}\right) / L\left(Y_{2}^{\prime}\right)
$$

in $S h v_{\text {Nis }}(\operatorname{SmCor}(k))$ induces an isomorphism $\mathbf{R} C(\pi)$ in $D M_{-}^{\text {eff }}(k)$.

Proof (see [VSF00, Proposition V.4.1.3] and its proof). The sequence

$$
0 \longrightarrow L\left(Y_{1}^{\prime}\right) \longrightarrow L\left(W_{1}\right) \oplus L\left(Y_{2}^{\prime}\right) \longrightarrow L\left(W_{2}\right)
$$

is exact and the quotient $L\left(W_{2}\right) /\left(L\left(W_{1}\right)+L\left(Y_{2}^{\prime}\right)\right)$ satisfies the condition of [VSF00, Theorem V.4.1.2].

\section{Analytical invariance}

Fix closed immersions $Y \hookrightarrow Y_{1}^{\prime} \hookrightarrow W_{1}$ and $Y \hookrightarrow Y_{2}^{\prime} \hookrightarrow W_{2}$ in $S c h^{\infty} / k$. Write $j_{\mathrm{m}}$ for the open immersion of $W_{\mathrm{m}}-Y_{\mathrm{m}}^{\prime}$, and $i_{Y, m}$ for the closed immersion of $Y$ into $W_{\mathrm{m}}$. The aim of this section is to prove the following.

Theorem 5.1 (Analytical invariance). Assume a given isomorphism

$$
f:\left(W_{1}\right)_{Y} \stackrel{\sim}{\longrightarrow}\left(W_{2}\right)_{Y}
$$

of formal completions along $Y$ inducing an isomorphism $\left(Y_{1}^{\prime}\right)_{Y} \cong\left(Y_{2}^{\prime}\right)_{Y}$, and compatible with the immersions $i_{Y, m}$ of $Y$. Then $f$ induces an isomorphism

$$
M_{\mathrm{gm}}\left(Y, i_{Y, 1}^{!} j_{1 !} \mathbb{Z}\right) \stackrel{\sim}{\longrightarrow} M_{\mathrm{gm}}\left(Y, i_{Y, 2}^{!} j_{2 !} \mathbb{Z}\right)
$$

in $D M_{-}^{\text {eff }}(k)$.

Remark 5.2. Using Proposition 2.4 together with Remark 3.3, we deduce the following statement from Theorem 5.1, assuming that $k$ admits resolution of singularities. Let $\bar{X}_{\mathrm{m}}$ be a compactification of $X_{\mathrm{m}} \in S c h / k, m=1,2$, and set $\partial \bar{X}_{\mathrm{m}}:=\bar{X}_{\mathrm{m}}-X_{\mathrm{m}}$ (with the reduced scheme structure). Assume that there is an isomorphism $\partial \bar{X}_{1} \cong \partial \bar{X}_{2}$, which can be extended to an isomorphism between the formal completions of $\bar{X}_{\mathrm{m}}$ along $\partial \bar{X}_{\mathrm{m}}$. Then $\partial M_{\mathrm{gm}}\left(X_{1}\right)$ and $\partial M_{\mathrm{gm}}\left(X_{2}\right)$ are isomorphic.

Note, however, that in practice it may not always be possible to identify the formal completion of $\partial \bar{X}$ along a given compactification $\bar{X}$ of a scheme $X$. Actually, one might control the formal completion of an abstract blow-up of each stratum belonging to a stratification of $\partial \bar{X}$. In order to compute $\partial M_{\mathrm{gm}}(X)$ in such a situation, one first applies co-localization with respect to the stratification, then uses invariance under abstract blow-up for each stratum, and finally analytical invariance as stated in the above generality.

The main technical ingredient of the proof of Theorem 5.1 is the following consequence of Artin approximation. 


\section{ThE BOUNDARY MOTIVE: DEFINITION AND BASIC PROPERTIES}

Theorem 5.3 (Artin). Let $S$ be the spectrum of a field or of an excellent Dedekind domain, let $W_{1}$ and $W_{2}$ be two $S$-schemes which are locally of finite type, let $Y_{\mathrm{m}}$ be closed sub-schemes of $W_{\mathrm{m}}$, $y_{\mathrm{m}} \in Y_{\mathrm{m}}$, and let $\mathfrak{a}_{\mathrm{m}}$ be the ideal of $Y_{\mathrm{m}}$ in the Henselization $\mathcal{O}_{W_{\mathrm{m}}, y_{\mathrm{m}}}^{\mathrm{h}}$ of the local ring $\mathcal{O}_{W_{\mathrm{m}}, y_{\mathrm{m}}}$. Denote by $\widehat{\mathcal{O}}_{W_{\mathrm{m}}, y_{\mathrm{m}}}$ the completion of $\mathcal{O}_{W_{\mathrm{m}}, y_{\mathrm{m}}}^{\mathrm{h}}$ with respect to $\mathfrak{a}_{\mathrm{m}}$, for $m=1,2$.

(a) If $\widehat{\mathcal{O}}_{W_{1}, y_{1}} \cong \widehat{\mathcal{O}}_{W_{2}, y_{2}}$ over $\mathcal{O}_{S}$, then $y_{1}$ and $y_{2}$ have a common Nisnevich neighbourhood: there exists an $S$-scheme $W^{\prime}$, a point $y^{\prime} \in W^{\prime}$ and étale morphisms $W^{\prime} \rightarrow W_{\mathrm{m}}$ mapping $y^{\prime}$ to $y_{\mathrm{m}}$, for $m=1,2$, which identify the residue fields $\kappa\left(y_{1}\right) \cong \kappa\left(y^{\prime}\right) \cong \kappa\left(y_{2}\right)$.

(b) Assume that in addition we are given an isomorphism $Y_{1} \cong Y_{2}$. Assume that the isomorphism in (a) maps the completed ideal $\hat{\mathfrak{a}}_{1}$ isomorphically to $\hat{\mathfrak{a}}_{2}$, and that the induced isomorphism $\mathcal{O}_{Y_{1}, y_{1}}^{\mathrm{h}} \cong \mathcal{O}_{Y_{2}, y_{2}}^{\mathrm{h}}$ is compatible with the given isomorphism $Y_{1} \cong Y_{2}$. Then the Nisnevich neighbourhood $W^{\prime}$ in (a) can be chosen such that in addition

$$
Y^{\prime}:=W^{\prime} \times_{W_{1}} Y_{1}=W^{\prime} \times_{W_{2}} Y_{2}
$$

as sub-schemes of $W^{\prime}$, and the induced étale morphisms $Y^{\prime} \rightarrow Y_{\mathrm{m}}$ are compatible with the isomorphism $Y_{1} \cong Y_{2}$.

Proof. This is a variant of [Art69, Corollary 2.6]. In fact, the results stated in [Art69, §2] are the translations of the main results of $\S 1$ only in the case when the ideal of definition is the maximal ideal of the point in question. In order to deduce the variant from [Art69, Theorem 1.12], one faithfully imitates the proof of [Art69, Corollary 2.6].

Corollary 5.4. With $S, W_{\mathrm{m}}$ and $Y_{\mathrm{m}}$ as in Theorem 5.3, assume a given isomorphism $Y_{1} \cong Y_{2}$ which extends to an isomorphism

$$
f:\left(W_{1}\right)_{Y_{1}} \stackrel{\sim}{\longrightarrow}\left(W_{2}\right)_{Y_{2}}
$$

of formal completions. Then there are Nisnevich coverings $\mathfrak{W}_{\mathrm{m}}$ of $W_{\mathrm{m}}$ of the form

$$
\mathfrak{W}_{1}=\left\{W_{i}^{\prime} \mid i \in I\right\} \coprod\left\{W_{1}-Y_{1}\right\}, \quad \mathfrak{W}_{2}=\left\{W_{i}^{\prime} \mid i \in I\right\} \coprod\left\{W_{2}-Y_{2}\right\}
$$

(with the same $W_{i}^{\prime}$ ) such that for any $i \in I$, one has

$$
Y_{i}^{\prime}:=W_{i}^{\prime} \times_{W_{1}} Y_{1}=W_{i}^{\prime} \times_{W_{2}} Y_{2}
$$

as sub-schemes of $W_{i}^{\prime}$, and the induced étale morphisms $Y_{i}^{\prime} \rightarrow Y_{\mathrm{m}}$ are compatible with the isomorphism $Y_{1} \cong Y_{2}$.

We turn to the proof of Theorem 5.1. Recall that $M_{\mathrm{gm}}\left(Y, i_{Y, \mathrm{~m}}^{!} j_{\mathrm{m} !} \mathbb{Z}\right)$ is associated to the following diagram.

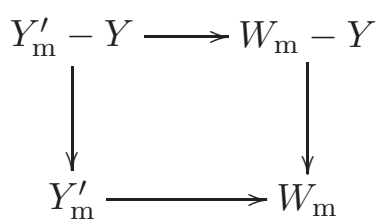

Since the two columns are of the same nature, Theorem 5.1 is a formal consequence of parts (a) and (b) of the following.

Theorem 5.5. Consider closed immersions $Y \hookrightarrow W_{1}$ and $Y \hookrightarrow W_{2}$ in $S c h^{\infty} / k$ which extend to an isomorphism

$$
f:\left(W_{1}\right)_{Y} \stackrel{\sim}{\longrightarrow}\left(W_{2}\right)_{Y}
$$

of formal completions along $Y$. 


\section{J. Wildeshaus}

(a) There is an isomorphism

$$
L\left(W_{1}\right) / L\left(W_{1}-Y\right) \stackrel{\sim}{\longrightarrow}\left(W_{2}\right) / L\left(W_{2}-Y\right)
$$

of Nisnevich sheaves with transfers, and depending only on $f$.

(b) The isomorphism $L\left(W_{1}\right) / L\left(W_{1}-Y\right) \stackrel{\sim}{\longrightarrow} L\left(W_{2}\right) / L\left(W_{2}-Y\right)$ is compatible with restriction of the $W_{\mathrm{m}}$ to sub-schemes containing $Y$.

(c) The isomorphism $L\left(W_{1}\right) / L\left(W_{1}-Y\right) \stackrel{\sim}{\longrightarrow} L\left(W_{2}\right) / L\left(W_{2}-Y\right)$ is compatible with restriction of $Y$.

Proof. Choose Nisnevich coverings

$$
\mathfrak{W}_{\mathrm{m}}=\left\{W_{i}^{\prime} \mid i \in I\right\} \coprod\left\{W_{\mathrm{m}}-Y\right\}
$$

as in Corollary 5.4. Set

$$
W^{\prime}:=\coprod_{i \in I} W_{i}^{\prime}
$$

and write $\alpha_{\mathrm{m}}$ for the coproduct of the étale morphisms from the $W_{i}^{\prime}$ to $W_{\mathrm{m}}$, for $m=1,2$. By Corollary 5.4, we have

$$
Y^{\prime}:=W^{\prime} \times_{W_{1}} Y=W^{\prime} \times_{W_{2}} Y
$$

and $\alpha_{1}$ and $\alpha_{2}$ coincide on $Y^{\prime}$. Using [VSF00, Proposition V.3.1.3], we see that we have exact sequences

$$
\begin{gathered}
\frac{L\left(W^{\prime} \times_{W_{1}} W^{\prime}\right)}{L\left(\left(W^{\prime}-Y^{\prime}\right) \times_{W_{1}}\left(W^{\prime}-Y^{\prime}\right)\right)} \stackrel{p r_{*}^{1}-p r_{*}^{2}}{\longrightarrow} \frac{L\left(W^{\prime}\right)}{L\left(W^{\prime}-Y^{\prime}\right)} \stackrel{\alpha_{1 *}}{\longrightarrow} \frac{L\left(W_{1}\right)}{L\left(W_{1}-Y\right)} \longrightarrow 0, \\
\frac{L\left(W^{\prime} \times_{W_{2}} W^{\prime}\right)}{L\left(\left(W^{\prime}-Y^{\prime}\right) \times_{W_{2}}\left(W^{\prime}-Y^{\prime}\right)\right)} \longrightarrow \frac{L\left(W^{\prime}\right)}{L\left(W^{\prime}-Y^{\prime}\right)} \stackrel{\alpha_{2 *}}{\longrightarrow} \frac{L\left(W_{2}\right)}{L\left(W_{2}-Y\right)} \longrightarrow 0,
\end{gathered}
$$

of Nisnevich sheaves with transfers. Let us show that the map $\alpha_{2 *}$ is zero on the image of $L\left(W^{\prime} \times_{W_{1}} W^{\prime}\right) / L\left(\left(W^{\prime}-Y^{\prime}\right) \times_{W_{1}}\left(W^{\prime}-Y^{\prime}\right)\right)$. We imitate the proof of [VSF00, Proposition II.4.3.9]. It is sufficient to show the following claim.

Claim 5.6. For any local Henselian scheme $S$ which is smooth over $k$, the composition

$$
c\left(S, W^{\prime} \times_{W_{1}} W^{\prime}\right) \stackrel{p r_{*}^{1}-p r_{*}^{2}}{\longrightarrow} c\left(S, W^{\prime}\right) \stackrel{\alpha_{2 *}}{\longrightarrow} \frac{c\left(S, W_{2}\right)}{c\left(S, W_{2}-Y\right)}
$$

is trivial.

Note that the presheaves $U \mapsto c(U, T)$, for $T \in S c h^{\infty} / k$, can be extended in an obvious way to the category of smooth $k$-schemes which are not necessarily of finite type. For the proof of Claim 5.6, we shall repeatedly apply the following principle, valid since $S$ is Henselian: for any $T \in S c h^{\infty} / k$, the support of any element of $c(S, T)$ is a disjoint union of local Henselian schemes. This principle allows us to consider only cycles in $c\left(S, W^{\prime} \times_{W_{1}} W^{\prime}\right)$ of the form $(Z)$, where $Z$ is a local Henselian sub-scheme of $S \times{ }_{k}\left(W^{\prime} \times_{W_{1}} W^{\prime}\right)$. Without loss of generality, we may assume that the closed point of $Z$ lies over $Y^{\prime}$, hence over $Y$. Write $p r_{*}^{l}(Z)=n^{l} \cdot\left(Z^{l}\right)$, with local Henselian sub-schemes $Z^{l}$ of $S \times_{k} W^{\prime}$, for $l=1,2$. We have $\alpha_{1 *} p r_{*}^{1}=\alpha_{1 *} p r_{*}^{2}$, hence the $\alpha_{1 *}\left(Z^{l}\right)$ are multiples of $\left(Z_{1}\right)$ for one local Henselian sub-scheme $\left(Z_{1}\right)$ of $S \times_{k} W_{1}$. In order to show the analogous statement for the $\alpha_{2 *}\left(Z^{l}\right)$, note first that the closed point $y$ of $Z_{1}$ belongs to $S \times_{k} Y$. The support of

$$
\alpha_{2 *}\left(p r_{*}^{1}-p r_{*}^{2}\right)(Z)=\alpha_{2 *}\left(n^{1} \cdot\left(Z^{1}\right)-n^{2} \cdot\left(Z^{2}\right)\right)
$$




\section{THE BOUNDARY MOTIVE: DEFINITION AND BASIC PROPERTIES}

is a disjoint union of local Henselian schemes, parametrized by their closed points. But since $\alpha_{1}$ and $\alpha_{2}$ coincide on $Y^{\prime}$, this support must be local, and we have indeed

$$
\alpha_{2 *}\left(p r_{*}^{1}-p r_{*}^{2}\right)(Z)=r \cdot\left(Z_{2}\right)
$$

for a local Henselian sub-scheme $\left(Z_{2}\right)$ of $S \times_{k} W_{2}$, whose closed point is $y$. In order to show that $r=0$, consider the commutative diagram

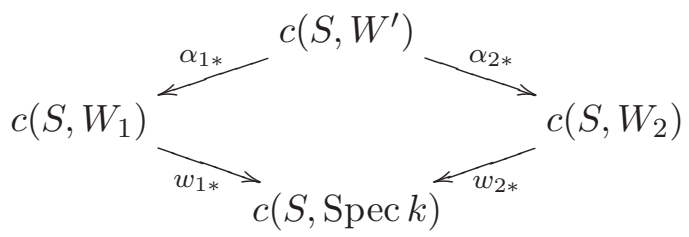

with $w_{\mathrm{m}}$ denoting the structure morphism of $W_{\mathrm{m}}$. On the one hand,

$$
w_{2 *} \alpha_{2 *}\left(p r_{*}^{1}-p r_{*}^{2}\right)(Z)=w_{1 *} \alpha_{1 *}\left(p r_{*}^{1}-p r_{*}^{2}\right)(Z)=w_{1 *}(0)=0 .
$$

On the other hand, $w_{2 *}\left(Z_{2}\right)$ is non-zero since $Z_{2}$ is finite over $S$. Hence $r$ must indeed be zero, and thus

$$
\alpha_{2 *}\left(p r_{*}^{1}-p r_{*}^{2}\right)(Z)=0 .
$$

This shows that $\alpha_{2 *}$ is zero on the image of $p r_{*}^{1}-p r_{*}^{2}$. By symmetry, we see that the identity on $L\left(W^{\prime}\right) / L\left(W^{\prime}-Y^{\prime}\right)$ factors to give an isomorphism

$$
L\left(W_{1}\right) / L\left(W_{1}-Y\right) \stackrel{\sim}{\longrightarrow}\left(W_{2}\right) / L\left(W_{2}-Y\right) .
$$

In order to prove that it does not depend on the choice of the Nisnevich coverings $\mathfrak{W}_{\mathrm{m}}$ as in Corollary 5.4, use the fact that the system of such coverings is filtering.

\section{Auto-duality}

Throughout this section, we assume that $k$ admits resolution of singularities. Under this assumption, $D M_{\mathrm{gm}}(k)$ is a rigid tensor triangulated category [VSF00, Theorem V.4.3.7 1 and 2]. In particular, there exists an internal Hom functor

$$
\underline{\text { Hom }}: D M_{\mathrm{gm}}(k) \times D M_{\mathrm{gm}}(k) \longrightarrow D M_{\mathrm{gm}}(k) .
$$

Writing $M^{*}:=\underline{\operatorname{Hom}}(M, \mathbb{Z}(0))$, we thus have $M=\left(M^{*}\right)^{*}$ for all $M \in D M_{\mathrm{gm}}(k)$.

Now fix $X \in S m / k$, and assume that $X$ is of pure dimension $n$. According to [VSF00, Theorem V.4.3.7 3], there is a canonical isomorphism

$$
\mu_{X}: M_{\mathrm{gm}}^{\mathrm{c}}(X) \stackrel{\sim}{\longrightarrow} M_{\mathrm{gm}}(X)^{*}(n)[2 n],
$$

hence by duality, a canonical isomorphism

$$
\nu_{X}:=\mu_{X}^{*}(n)[2 n]: M_{\mathrm{gm}}(X) \stackrel{\sim}{\longrightarrow} M_{\mathrm{gm}}^{\mathrm{c}}(X)^{*}(n)[2 n] .
$$

The aim of this section is to prove the following.

Theorem 6.1 (Auto-duality). There exists a canonical isomorphism

$$
\eta_{X}: \partial M_{\mathrm{gm}}(X) \stackrel{\sim}{\longrightarrow} \partial M_{\mathrm{gm}}(X)^{*}(n)[2 n-1] .
$$




\section{J. Wildeshaus}

It fits into the following morphism of exact triangles.

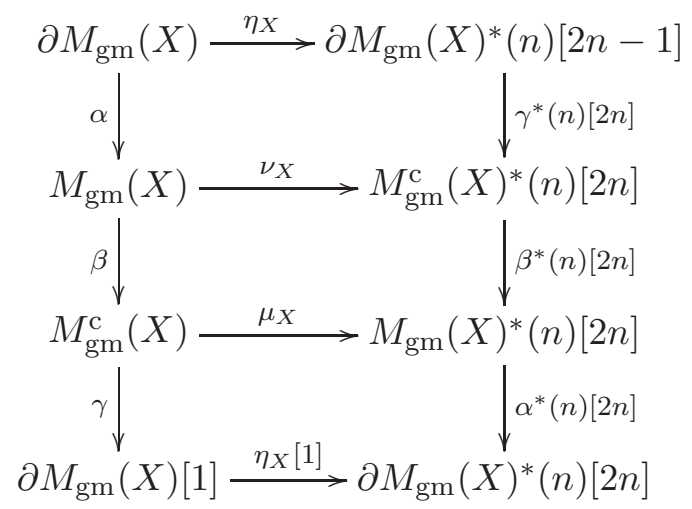

Furthermore, it is itself auto-dual in the sense that the equality

$$
\eta_{X}=\eta_{X}^{*}(n)[2 n-1]
$$

holds.

First observe that the existence of some isomorphism $\eta_{X}$ fitting into the above diagram of exact triangles is a consequence of the axioms of triangulated categories, and the commutativity of the following diagram.

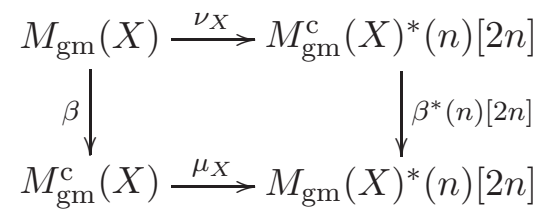

Thus, the point of auto-duality is that $\eta_{X}$ can be defined canonically, and that this definition is itself auto-dual.

For the proof of Theorem 6.1, observe that by adjunction, the construction of $\eta_{X}$ is equivalent to the construction of a pairing

$$
(\bullet, \bullet): \partial M_{\mathrm{gm}}(X) \otimes \partial M_{\mathrm{gm}}(X)[1] \longrightarrow \mathbb{Z}(n)[2 n] .
$$

We are thus led to investigate morphisms in $D M_{\mathrm{gm}}^{\mathrm{eff}}(k)$ whose target is $\mathbb{Z}(n)[2 n]$. The statement we are aiming for is Theorem 6.14. It is a generalization of [VSF00, Corollary V.4.2.5]. In the above geometric context, it implies that certain codimension $n$-cycles on the self-product $\bar{X} \times_{k} \bar{X}$, where $\bar{X}$ is a smooth compactification of $X$, yield morphisms of the type of $(\bullet, \bullet)$. In order to prepare Theorem 6.14, we need to prove a variant of duality for bivariant cycle cohomology (Theorem 6.11). Let us start by recalling the definition of certain variants of the Nisnevich sheaves with transfers $L^{\mathrm{c}}(V)=z(\bullet, V)($ see $[\mathrm{VSF} 00$, p. 228]).

Definition 6.2. Let $V \in S c h / k, W \in S m / k$ and $r \geqslant 0$.

(a) The Nisnevich sheaf with transfers $z_{\text {equi }}(V, r)$ associates to $U \in S m / k$ the free Abelian group on the symbols $(Z)$, where $Z$ runs through the integral closed sub-schemes of $U \times_{k} V$ which are equi-dimensional of relative dimension $r$ over $U$ and dominant over a connected component of $U$.

(b) The Nisnevich sheaf with transfers $z_{\text {equi }}(W, V, r)$ maps $U \in S m / k$ to $z_{\text {equi }}(V, r)\left(U \times_{k} W\right)$.

Note that the sheaves $z_{\text {equi }}(W, V, r)$ are contravariant in the first variable. Now recall [VSF00, Corollary V.4.1.8]. 


\section{THE BOUNDARY MOTIVE: DEFINITION AND BASIC PROPERTIES}

Proposition 6.3. The object $\mathbb{Z}(n)[2 n]$ of $D M_{\mathrm{gm}}^{\mathrm{eff}}(k) \subset D M_{-}^{\mathrm{eff}}(k)$ is represented by the complex

$$
M_{\mathrm{gm}}^{\mathrm{c}}\left(\mathbb{A}^{n}\right)=\underline{C}_{*}\left(z\left(\bullet, \mathbb{A}^{n}\right)\right)=\underline{C}_{*}\left(z_{\text {equi }}\left(\mathbb{A}^{n}, 0\right)\right) .
$$

More generally, if $W \in S m / k$, then the complex

$$
\underline{C}_{*}\left(z_{\text {equi }}\left(W, \mathbb{A}^{n}, 0\right)\right)
$$

represents the functor

$$
\underline{H o m}_{D M_{-}^{\text {eff }}(k)}\left(M_{\mathrm{gm}}(W), \mathbb{Z}(n)[2 n]\right)
$$

on $D M_{-}^{\text {eff }}(k)$ [VSF00, Corollary V.4.2.7]. We need a variant of this statement as follows.

Definition 6.4. Let $V \in S c h / k, W \in S m / k$ and $r \geqslant 0$. Assume further that $Y \subset W$ is a closed sub-scheme.

(a) Define

$$
M_{\mathrm{gm}}(W / Y):=\underline{C}_{*}(L(Y \rightarrow W)),
$$

where $L(Y \rightarrow W)$ is the complex given by $L(Y)$ in degree -1 and $L(W)$ in degree zero, the differential being induced by the immersion of $Y$.

(b) Assume in addition that arbitrary intersections of the components $Y_{j}$ of $Y$ are smooth. Define

$$
z_{\text {equi }}\left(Y^{\bullet} \rightarrow W, V, r\right)
$$

as the complex of Nisnevich sheaves with transfers whose zeroth component is $z_{\text {equi }}(W, V, r)$, and whose $m$ th component, for $m \geqslant 1$, is the direct sum of the $z_{\text {equi }}\left(Y_{J}, V, 0\right)$, for all $m$-fold intersections $Y_{J}$ of the $Y_{j}$. The differentials are induced by contravariance of the sheaves $z_{\text {equi }}(\bullet, V, r)$.

Of course, $M_{\mathrm{gm}}(W / Y)$ is the same thing as $M_{\mathrm{gm}}$ evaluated at the diagram

$$
Y \longrightarrow W
$$

(see Convention 1.2).

Proposition 6.5. Let $W \in S m / k$. Assume further that $Y \subset W$ is a closed sub-scheme such that arbitrary intersections of the components of $Y$ are smooth. Then the complex

$$
\underline{C}_{*}\left(z_{\text {equi }}\left(Y^{\bullet} \rightarrow W, \mathbb{A}^{n}, 0\right)\right)
$$

represents the functor

$$
\underline{H o m}_{D M_{-}^{\text {eff }}(k)}\left(M_{\mathrm{gm}}(W / Y), \mathbb{Z}(n)[2 n]\right)
$$

on $D M_{-}^{\mathrm{eff}}(k)$. This identification is compatible with passage from the pair $Y \subset W$ to $Y^{\prime} \subset U$, for open sub-schemes $U$ of $W$, and closed sub-schemes $Y^{\prime}$ of $Y \cap U$ such that arbitrary intersections of the components $Y_{j}^{\prime}$ of $Y^{\prime}$ are smooth.

Convention 6.6. By over-simplification of language, we shall refer to the last compatibility statement in Proposition 6.5 as 'compatibility with restriction of $W$ and $Y$ '.

Proof of Proposition 6.5. First, observe that the canonical morphism $M_{\mathrm{gm}}\left(Y^{\bullet}\right) \rightarrow M_{\mathrm{gm}}(Y)$ is an isomorphism. This follows from induction on the number of components $Y_{j}$. The induction step is provided by [VSF00, Proposition V.4.1.3]. Similarly, $M_{\mathrm{gm}}\left(Y^{\bullet} \rightarrow W\right) \rightarrow M_{\mathrm{gm}}(W / Y)$ is an isomorphism.

As in the proof of [VSF00, Proposition V.4.2.8], one has a canonical morphism of complexes can from $\underline{C}_{*}\left(z_{\text {equi }}\left(Y^{\bullet} \rightarrow W, \mathbb{A}^{n}, 0\right)\right)=\underline{C}_{*}\left(p_{*} p^{*}\left(L^{\mathrm{c}}\left(\mathbb{A}^{n}\right)\right)\right)$ to $R p_{*}\left(p^{*} \underline{C}_{*}\left(L^{\mathrm{c}}\left(\mathbb{A}^{n}\right)\right)\right)$, where $p$ denotes 


\section{J. Wildeshaus}

the structure morphism of the diagram $Y^{\bullet} \rightarrow W$. By an obvious generalization of the last part of [VSF00, Proposition V.3.2.8], one has

$$
R p_{*}\left(p^{*} \underline{C}_{*}\left(L^{\mathrm{c}}\left(\mathbb{A}^{n}\right)\right)\right)=\underline{\operatorname{Hom}}\left(M_{\mathrm{gm}}\left(Y^{\bullet} \rightarrow W\right), \mathbb{Z}(n)[2 n]\right)
$$

in $D M_{-}^{\text {eff }}(k)$. To check that can is an isomorphism, one uses the spectral sequences on both its source and target, associated to the stupid filtration (filtration bête) of $Y^{\bullet} \rightarrow W$. Result [VSF00, Corollary V.4.2.7] shows that can is an isomorphism on the $E_{1}$-terms of this spectral sequence.

Remark 6.7. In the situation of Proposition 6.5, assume in addition that $W$ is of dimension at most $n$. By [VSF00, Corollary V.4.3.6], the object $M_{\mathrm{gm}}(W / Y)^{*}(n)[2 n]$ of $D M_{\mathrm{gm}}(k)$ belongs to $D M_{\mathrm{gm}}^{\mathrm{eff}}(k)$, and its image under the embedding into $D M_{-}^{\text {eff }}(k)$ equals $\underline{H o m}_{D M_{-}^{\text {eff }}(k)}\left(M_{\mathrm{gm}}(W / Y)\right.$, $\mathbb{Z}(n)[2 n])$. It is thus represented by the complex $\underline{C}_{*}\left(z_{\text {equi }}\left(Y^{\bullet} \rightarrow W, \mathbb{A}^{n}, 0\right)\right)$.

Given an object $F$ of $S h v_{\text {Nis }}(\operatorname{SmCor}(k))$, denote by $\underline{h}^{l}(F)$ the $l$ th cohomology object of the complex $\underline{C}_{*}(F)$. Thus, the lth cohomology object of $\underline{C}_{*}\left(z_{\text {equi }}\left(Y^{\bullet} \rightarrow W, \mathbb{A}^{n}, 0\right)\right)($ Spec $k)$ equals $\underline{h}^{l}\left(z_{\text {equi }}\left(Y^{\bullet} \rightarrow W, \mathbb{A}^{n}, 0\right)\right)(\operatorname{Spec} k)$.

Corollary 6.8. In the situation of Proposition 6.5, there is a canonical isomorphism

$$
c_{W / Y}: \underline{h}^{l}\left(z_{\text {equi }}\left(Y^{\bullet} \rightarrow W, \mathbb{A}^{n}, 0\right)\right)(\operatorname{Spec} k) \stackrel{\sim}{\longrightarrow} \operatorname{Hom}\left(M_{\mathrm{gm}}(W / Y), \mathbb{Z}(n)[2 n+l]\right) .
$$

Here Hom denotes morphisms in $D M_{\mathrm{gm}}^{\mathrm{eff}}(k)$. The isomorphism is compatible with restriction of $W$ and $Y$ in the sense of Convention 6.6.

Proof. First, we have

$$
\operatorname{Hom}\left(M_{\mathrm{gm}}(W / Y), \mathbb{Z}(n)[2 n+l]\right)=\operatorname{Hom}_{D M_{-}^{\mathrm{eff}}(k)}\left(M_{\mathrm{gm}}(W / Y), \mathbb{Z}(n)[2 n+l]\right)
$$

since the embedding of $D M_{\mathrm{gm}}^{\mathrm{eff}}(k)$ into $D M_{-}^{\mathrm{eff}}(k)$ is full. Adjointness of $\otimes$ and $\underline{H o m}$ implies that $\operatorname{Hom}_{D M_{-}^{\text {eff }}(k)}\left(M_{\mathrm{gm}}(W / Y), \mathbb{Z}(n)[2 n+l]\right)$ equals

$$
\operatorname{Hom}_{D M_{-}^{\text {eff }}(k)}\left(M_{\mathrm{gm}}(\operatorname{Spec} k), \underline{\operatorname{Hom}}_{D M_{-}^{\mathrm{eff}}(k)}\left(M_{\mathrm{gm}}(W / Y), \mathbb{Z}(n)[2 n+l]\right)\right) .
$$

By Proposition 6.5, this group equals

$$
\operatorname{Hom}_{D M_{-}^{\text {eff }}(k)}\left(\mathbf{R} C(L(\operatorname{Spec} k)), \mathbf{R} C\left(z_{\text {equi }}\left(Y^{\bullet} \rightarrow W, \mathbb{A}^{n}, 0\right)\right)[l]\right) .
$$

Now use the fact that $\mathbf{R} C$ is left adjoint to the inclusion of $D M_{-}^{\text {eff }}(k)$ into $D^{-}\left(\operatorname{Sh} v_{\mathrm{Nis}}(\operatorname{SmCor}(k))\right)$.

For later use, we also note a consequence of the special case $Y=\emptyset$.

Corollary 6.9. Let $W \in S m / k$. Then the functor on open sub-schemes of $W$,

$$
U \longmapsto \underline{C}_{*}\left(z_{\text {equi }}\left(U, \mathbb{A}^{n}, 0\right)\right),
$$

satisfies the Mayer-Vietoris property in the following sense. Given an equality $U=U_{1} \cup U_{2}$ of open sub-schemes of $W$, the exact sequence

$$
0 \rightarrow z_{\text {equi }}\left(U, \mathbb{A}^{n}, 0\right) \rightarrow z_{\text {equi }}\left(U_{1}, \mathbb{A}^{n}, 0\right) \oplus z_{\text {equi }}\left(U_{2}, \mathbb{A}^{n}, 0\right) \rightarrow z_{\text {equi }}\left(U_{1} \cap U_{2}, \mathbb{A}^{n}, 0\right)
$$

in $S h v_{\mathrm{Nis}}(\operatorname{SmCor}(k))$ induces an exact triangle

$$
\begin{aligned}
\mathbf{R} C\left(z_{\text {equi }}\left(U, \mathbb{A}^{n}, 0\right)\right) \longrightarrow \mathbf{R} C\left(z_{\text {equi }}\left(U_{1}, \mathbb{A}^{n}, 0\right)\right) \oplus \mathbf{R} C\left(z_{\text {equi }}\left(U_{2}, \mathbb{A}^{n}, 0\right)\right) & \\
\longrightarrow \mathbf{R} C\left(z_{\text {equi }}\left(U_{1} \cap U_{2}, \mathbb{A}^{n}, 0\right)\right) & \longrightarrow \mathbf{R} C\left(z_{\text {equi }}\left(U, \mathbb{A}^{n}, 0\right)\right)[1]
\end{aligned}
$$

in $D M_{-}^{\mathrm{eff}}(k)$. 


\section{THE BOUNDARY MOTIVE: DEFINITION AND BASIC PROPERTIES}

Proof. This follows from Proposition 6.5 and the Mayer-Vietoris property for the functor $U \mapsto$ $M_{\mathrm{gm}}(U)$ [VSF00, Proposition V.4.1.1].

We need to find a way to efficiently generate elements in the group

$$
\underline{h}^{0}\left(z_{\text {equi }}\left(Y^{\bullet} \rightarrow W, \mathbb{A}^{n}, 0\right)\right)(\operatorname{Spec} k) .
$$

Definition 6.10. Let $W \in S m / k$, and assume that $W$ is of pure dimension $m$. Fix a closed subscheme $Y \subset W$ and an object $V$ in $S c h / k$. Let $r \geqslant 0$. Define the sub-sheaf

$$
z_{\text {equi }}\left(W \times_{k} V, m+r\right)_{Y} \subset z_{\text {equi }}\left(W \times_{k} V, m+r\right)
$$

as follows. Map $U \in S m / k$ to the free Abelian group on the symbols $(Z)$, where $Z$ runs through those generators of $z_{\text {equi }}\left(W \times_{k} V, m+r\right)(U)$ such that for any geometric point

$$
\operatorname{Spec}(\bar{k}) \longrightarrow U \times_{k} Y \longleftrightarrow U \times_{k} W
$$

of $U \times_{k} Y$, the sub-scheme

$$
Z \times_{U \times{ }_{k} W} \operatorname{Spec}(\bar{k})
$$

of $V_{\bar{k}}$ is empty or of dimension $r$. The sheaf of Abelian monoids

$$
z_{\text {equi }}^{\text {eff }}\left(W \times_{k} V, m+r\right)_{Y} \subset z_{\text {equi }}\left(W \times_{k} V, m+r\right)_{Y}
$$

is defined as the intersection of $z_{\text {equi }}\left(W \times{ }_{k} V, m+r\right)_{Y}$ with the monoid of effective cycles $z_{\text {equi }}^{\text {eff }}\left(W \times_{k} V\right.$, $m+r)$ in $z_{\text {equi }}\left(W \times_{k} V, m+r\right)$.

One checks that $z_{\text {equi }}\left(W \times_{k} V, m+r\right)_{Y}$ and $z_{\text {equi }}^{\text {eff }}\left(W \times_{k} V, m+r\right)_{Y}$ inherit the transfers from $z_{\text {equi }}\left(W \times{ }_{k} V, m+r\right)$. If one imposes the defining condition on all geometric points of $U \times{ }_{k} W$ instead of just those of $U \times_{k} Y$, then one obtains $z_{\text {equi }}(W, V, r)(U)$. Hence $z_{\text {equi }}(W, V, r)$ is a sub-sheaf of $z_{\text {equi }}\left(W \times_{k} V, m+r\right)_{Y}$ :

$$
\mathcal{D}: z_{\text {equi }}(W, V, r) \longleftrightarrow z_{\text {equi }}\left(W \times_{k} V, m+r\right)_{Y} .
$$

Define the natural inclusion

$$
\iota: z_{\text {equi }}\left(W \times_{k} V, m+r\right)_{Y} \longleftrightarrow z_{\text {equi }}\left(W \times_{k} V, m+r\right) .
$$

The Moving Lemma [VSF00, Theorem IV.6.3] implies (see [VSF00, Lemma IV.6.6]) that if both $W$ and $V$ are smooth and projective, then both $\mathbf{R} C(\iota)$ and $\mathbf{R} C(\iota) \circ \mathbf{R} C(\mathcal{D})$ are isomorphisms. Hence $\mathbf{R} C(\mathcal{D})$ is an isomorphism if both $W$ and $V$ are smooth and projective. Our aim is to prove this statement under less restrictive hypotheses on $W$ and $V$. Our result is a variant of duality for bivariant cycle cohomology [VSF00, Theorem IV.7.4].

Theorem 6.11. Let $W \in S m / k$ be quasi-projective and of pure dimension $m$. Let $Y \subset W$ be a closed sub-scheme and $V \in S c h / k$. Let $r \geqslant 0$. Then the inclusion

$$
\mathcal{D}: z_{\text {equi }}(W, V, r) \longleftrightarrow z_{\text {equi }}\left(W \times_{k} V, m+r\right)_{Y}
$$

induces an isomorphism $\mathbf{R} C(\mathcal{D})$. It is compatible with restriction of $W$ and $Y$ in the sense of Convention 6.6.

Proof. We shall follow faithfully the strategy of [VSF00, pp. 172-176]. Fix compactifications $\bar{W}$ of $W$ and $\bar{V}$ of $V$ with a smooth and projective $\bar{W}$. For any proper $\bar{V}$-scheme $\bar{T}$, define the morphism

$$
\alpha_{\bar{T}}: z_{\text {equi }}\left(\bar{W} \times_{k} \bar{T}, m+r\right) \longrightarrow z_{\text {equi }}\left(\bar{W} \times_{k} \bar{V}, m+r\right) \longrightarrow z_{\text {equi }}\left(W \times_{k} V, m+r\right)
$$

as the composition of proper push-forward with restriction [VSF00, pp. 141-142]. Similarly, define the variant on effective cycle sheaves

$$
\alpha \alpha_{\bar{T}}^{\text {eff }}: z_{\text {equi }}^{\text {eff }}\left(\bar{W} \times_{k} \bar{T}, m+r\right) \longrightarrow z_{\text {equi }}^{\text {eff }}\left(W \times_{k} V, m+r\right) .
$$




\section{J. Wildeshaus}

Denote by $\Psi_{\bar{T}} \subset z_{\text {equi }}\left(\bar{W} \times_{k} \bar{T}, m+r\right)$ the sub-sheaf of Abelian groups generated by $\left(\alpha \frac{\text { eff }}{\bar{T}}\right)^{-1}$ $\left(z_{\text {equi }}^{\text {eff }}\left(W \times_{k} V, m+r\right)_{Y}\right)$. We have

$$
\Psi_{\bar{V}}=\alpha_{\bar{V}}^{-1}\left(z_{\text {equi }}\left(W \times_{k} V, m+r\right)_{Y}\right) .
$$

We claim that the inclusion

$$
\iota_{\bar{T}}: \Psi_{\bar{T}} \longleftrightarrow z_{\text {equi }}\left(\bar{W} \times_{k} \bar{T}, m+r\right)
$$

induces a quasi-isomorphism $\underline{C}_{*}(\iota \bar{T})$. In order to prove this claim, imitate the proof of [VSF00, Proposition IV.7.3]. One uses the Moving Lemma that we have already cited. It is here that the projectivity assumption on $\bar{W}$ enters.

Next, one imitates the proof of [VSF00, Theorem IV.7.4], using the above instead of [VSF00, Proposition IV.7.3], to see that the inclusion

$$
\iota: z_{\text {equi }}\left(W \times_{k} V, m+r\right)_{Y} \longleftrightarrow z_{\text {equi }}\left(W \times_{k} V, m+r\right)
$$

induces a quasi-isomorphism $\underline{C}_{*}(\iota)$. The same observation, applied to the case where $Y=W$ implies that the composition $\mathbf{R} C(\iota) \circ \mathbf{R} C(\mathcal{D})$ is an isomorphism. (This is, of course, the original statement of [VSF00, Theorem IV.7.4].)

We unite the assumptions from Proposition 6.5 and Definition 6.10 . Hence $W \in S m / k$ is of pure dimension $m$, and $Y \subset W$ is a closed sub-scheme such that arbitrary intersections of the components of $Y$ are smooth. Observe that the condition on elements in $z_{\text {equi }}\left(W \times_{k} \mathbb{A}^{n}, m\right)_{Y}$ ensures, in particular, that they intersect properly with the components $Y_{j} \times{ }_{k} \mathbb{A}^{n}$ of $Y \times_{k} \mathbb{A}^{n}$. This allows us to define an inverse image $\delta$ making the following diagram commutative.

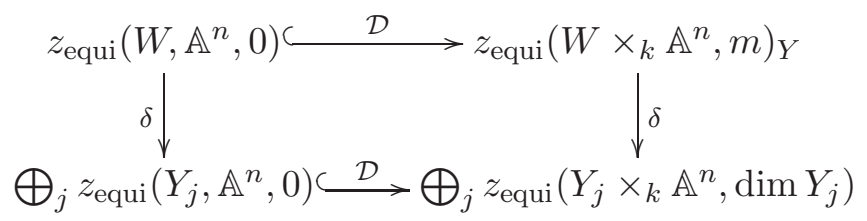

The group in the lower right-hand corner has to be modified if $Y_{j}$ has several components of different dimension. But in fact, $z_{\text {equi }}\left(W \times_{k} \mathbb{A}^{n}, m\right)_{Y}$ is defined such that $\delta$ not only exists but maps $z_{\text {equi }}\left(W \times_{k} \mathbb{A}^{n}, m\right)_{Y}$ to $\oplus_{j} z_{\text {equi }}\left(Y_{j}, \mathbb{A}^{n}, 0\right)$. Thus we may enlarge the complex

$$
z_{\text {equi }}\left(Y^{\bullet} \rightarrow W, \mathbb{A}^{n}, 0\right)
$$

by replacing its zeroth component $z_{\text {equi }}\left(W, \mathbb{A}^{n}, 0\right)$ with $z_{\text {equi }}\left(W \times_{k} \mathbb{A}^{n}, m\right)_{Y}$ :

$$
z_{\text {equi }}\left(Y^{\bullet} \rightarrow W, \mathbb{A}^{n}, 0\right) \subset z_{\text {equi }}\left(Y^{\bullet} \rightarrow W, \mathbb{A}^{n}, 0\right)^{\prime} .
$$

When $W$ is quasi-projective, then Theorem 6.11 shows that this inclusion of complexes induces an isomorphism after application of $\mathbf{R} C$. In the general case, there is a (mainly notational) complication since we do not know whether the functor on open sub-schemes of $W$,

$$
U \longmapsto \underline{C}_{*}\left(z_{\text {equi }}\left(U \times_{k} \mathbb{A}^{n}, m\right)_{Y}\right),
$$

satisfies the Mayer-Vietoris property. Therefore, we fix an additional geometric datum, namely a finite open covering $\left\{W_{\alpha}\right\}$ of $W$ by quasi-projective schemes. Consider the covering of $Y$ induced by $\left\{W_{\alpha}\right\}$, and define

$$
z_{\text {equi }}\left(Y^{\bullet} \rightarrow W, \mathbb{A}^{n}, 0\right)^{\prime \prime}
$$

as the simple complex of the double Čech complex associated to this covering and $z_{\text {equi }}\left(Y^{\bullet} \rightarrow W\right.$, $\left.\mathbb{A}^{n}, 0\right)^{\prime}$. For example, the components of degree 0 and 1 are

$$
\bigoplus_{\alpha} z_{\text {equi }}\left(W_{\alpha} \times{ }_{k} \mathbb{A}^{n}, m\right)_{Y}
$$


and

$$
\bigoplus_{\alpha, j} z_{\text {equi }}\left(W_{\alpha} \cap Y_{j}, \mathbb{A}^{n}, 0\right) \oplus \bigoplus_{\alpha_{1} \neq \alpha_{2}} z_{\text {equi }}\left(\left(W_{\alpha_{1}} \cap W_{\alpha_{2}}\right) \times_{k} \mathbb{A}^{n}, m\right)_{Y}
$$

Consider the natural inclusion

$$
\mathcal{D}: z_{\text {equi }}\left(Y^{\bullet} \rightarrow W, \mathbb{A}^{n}, 0\right) \longleftrightarrow z_{\text {equi }}\left(Y^{\bullet} \rightarrow W, \mathbb{A}^{n}, 0\right)^{\prime},
$$

and use the same symbol for the composition of $\mathcal{D}$ with the co-augmentation

$$
z_{\text {equi }}\left(Y^{\bullet} \rightarrow W, \mathbb{A}^{n}, 0\right)^{\prime} \longrightarrow z_{\text {equi }}\left(Y^{\bullet} \rightarrow W, \mathbb{A}^{n}, 0\right)^{\prime \prime}
$$

Corollary 6.12. The morphism

$$
\mathcal{D}: z_{\text {equi }}\left(Y^{\bullet} \rightarrow W, \mathbb{A}^{n}, 0\right) \longrightarrow z_{\text {equi }}\left(Y^{\bullet} \rightarrow W, \mathbb{A}^{n}, 0\right)^{\prime \prime}
$$

induces an isomorphism $\mathbf{R} C(\mathcal{D})$. In particular, different choices of coverings $W=\cup_{\alpha} W_{\alpha}$ give rise to the same object $\mathbf{R} C\left(z_{\text {equi }}\left(Y^{\bullet} \rightarrow W, \mathbb{A}^{n}, 0\right)^{\prime \prime}\right)$. The isomorphism $\mathbf{R} C(\mathcal{D})$ is compatible with restriction of $W$ and $Y$ in the sense of Convention 6.6.

Proof. This follows from Theorem 6.11 and Corollary 6.9.

Definition 6.13. Define the sub-sheaf with transfers

$$
z_{\text {equi }}(W, m-n)_{Y} \subset z_{\text {equi }}(W, m-n)
$$

as the sub-sheaf of cycles having empty intersection with $Y$.

Note that $z_{\text {equi }}(W, m-n)_{Y}$ behaves contravariantly with respect to restriction of $W$ and $Y$. Flat pull-back defines a morphism

$$
p_{\mathbb{A}^{n}}^{*}: z_{\text {equi }}(W, m-n)_{Y}[0] \longrightarrow z_{\text {equi }}\left(Y^{\bullet} \rightarrow W, \mathbb{A}^{n}, 0\right)^{\prime} .
$$

Its composition with the co-augmentation

$$
z_{\text {equi }}\left(Y^{\bullet} \rightarrow W, \mathbb{A}^{n}, 0\right)^{\prime} \longrightarrow z_{\text {equi }}\left(Y^{\bullet} \rightarrow W, \mathbb{A}^{n}, 0\right)^{\prime \prime}
$$

induces a morphism on the level of $\underline{h}^{0}(\operatorname{Spec} k)$, denoted by the same symbol $p_{\mathbb{A}^{n}}^{*}$. Putting everything together, we obtain the following.

Theorem 6.14. Let $W \in S m / k$ be of pure dimension $m$ and let $Y \subset W$ be a closed sub-scheme such that arbitrary intersections of the components of $Y$ are smooth. Then there is a unique morphism

$$
\operatorname{cyc}_{W / Y}: \underline{h}^{0}\left(z_{\text {equi }}(W, m-n)_{Y}\right)(\operatorname{Spec} k) \longrightarrow \operatorname{Hom}\left(M_{\mathrm{gm}}(W / Y), \mathbb{Z}(n)[2 n]\right)
$$

making the following diagram commute.

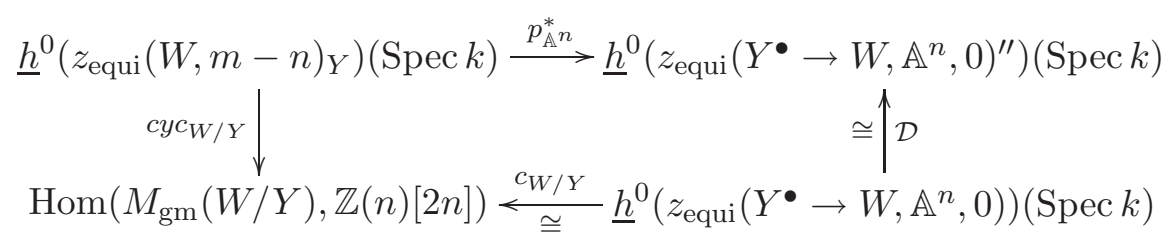

Here Hom denotes morphisms in $D M_{\mathrm{gm}}^{\mathrm{eff}}(k)$. The morphism $c y c_{W / Y}$ is compatible with restriction of $W$ and $Y$ in the sense of Convention 6.6.

Proof. Apply Corollaries 6.8 and 6.12 .

Remark 6.15. Another type of compatibility property with respect to change of $W$ and $Y$ is useful. Assume a given second pair $Y_{1} \subset W_{1}$ of schemes satisfying the hypotheses of Theorem 6.14 , and 


\section{J. Wildeshaus}

a proper morphism $W_{1} \rightarrow W$ identifying $Y_{1}$ with the fibre product $W_{1} \times_{W} Y$, and inducing an isomorphism from $W_{1}-Y_{1}$ to $W-Y$. Theorem 4.2 tells us that

$$
M_{\mathrm{gm}}\left(W_{1} / Y_{1}\right) \longrightarrow M_{\mathrm{gm}}(W / Y)
$$

is an isomorphism. On the other hand, we clearly have

$$
z_{\text {equi }}(W, m-n)_{Y}=z_{\text {equi }}\left(W_{1}, m-n\right)_{Y_{1}} .
$$

It is easy to see that in this situation, the diagram

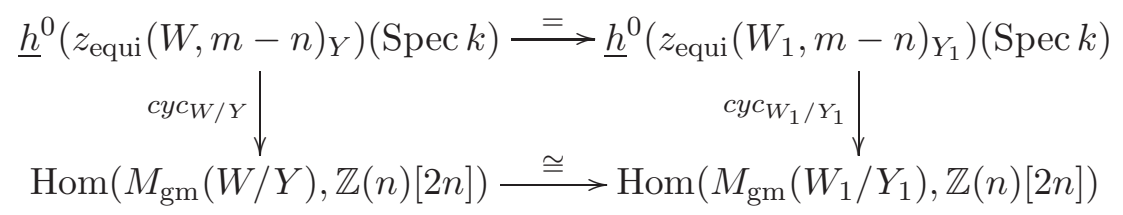

commutes.

When $Y$ is empty, then [VSF00, Corollary V.4.2.5] tells us that $c y c_{W}:=c y c_{W / \emptyset}$ is an isomorphism. We have not attempted to prove whether the analogous statement for $c y c_{W / Y}$ is true when $Y$ is non-empty.

Proof of Theorem 6.1. We start by introducing the notation $\operatorname{Cyc}^{n}(W)_{Y}$ for the group $z_{\text {equi }}(W$, $m-n)_{Y}(\operatorname{Spec} k)$, when $W$ and $Y \subset W$ are as before. By definition, $C y c^{n}(W)_{Y}$ is the group of codimension $n$ cycles on $W$ not meeting $Y$. Write $C y c^{n}(W)$ for the group of all codimension $n$ cycles on $W$. Our proof relies on the following principles, which are consequences of Theorem 6.14 and of the definition of the tensor structure on $D M_{-}^{\text {eff }}(k)$.

(A) For $i=1,2$, let $W_{i} \in S m / k$ be of pure dimension, with closed sub-schemes $Y_{i} \subset W_{i}$, such that arbitrary intersections of the components of the $Y_{i}$ are smooth. Then any

$$
c \in C y c^{n}\left(W_{1} \times_{k} W_{2}\right)_{Y_{1} \times_{k} W_{2} \cup W_{1} \times_{k} Y_{2}}
$$

defines a pairing

$$
(\bullet, \bullet)_{c}: M_{\mathrm{gm}}\left(W_{1} / Y_{1}\right) \otimes M_{\mathrm{gm}}\left(W_{2} / Y_{2}\right) \longrightarrow \mathbb{Z}(n)[2 n],
$$

or equivalently, a morphism

$$
\varepsilon_{c}: M_{\mathrm{gm}}\left(W_{1} / Y_{1}\right) \longrightarrow M_{\mathrm{gm}}\left(W_{2} / Y_{2}\right)^{*}(n)[2 n]
$$

in $D M_{\mathrm{gm}}(k)$. The morphism $\varepsilon_{c}$ is induced by a morphism of Nisnevich sheaves

$$
e_{c^{\prime}}: c\left(\bullet, W_{1}\right) / c\left(\bullet, Y_{1}\right) \longrightarrow z_{\text {equi }}\left(Y_{2}^{\bullet} \rightarrow W_{2}, \mathbb{A}^{n}, 0\right)
$$

defined as follows. Use Corollary 6.12 to move the pull-back $p_{\mathbb{A}^{n}}^{*}(c)$ into a cycle

$$
c^{\prime} \in z_{\text {equi }}\left(W_{1} \times_{k} W_{2}, \mathbb{A}^{n}, 0\right)(\operatorname{Spec} k)
$$

having empty intersection with $Y_{1} \times_{k} W_{2} \cup W_{1} \times_{k} Y_{2}$. Given $U \in S m / k$ and $\mathcal{Z} \in c\left(U, W_{1}\right)$, pull back $\mathcal{Z}$ to $W_{2}$, giving $\mathcal{Z}_{W_{2}} \in c\left(U \times_{k} W_{2}, W_{1}\right)$. Similarly, pull back $c^{\prime}$ to $U$, giving $c_{U}^{\prime}$. Now consider the cycle

$$
c^{\prime} \cap \mathcal{Z}_{W_{2}}:=\operatorname{Cor}_{W_{1} \times_{k} W_{2} \times_{k} \mathbb{A}^{n} / W_{1} \times_{k} W_{2}}\left(c_{U}^{\prime} \otimes \mathcal{Z}_{W_{2}}\right)
$$

where $\operatorname{Cor}_{W_{1} \times_{k} W_{2} \times{ }_{k} \mathbb{A}^{n} / W_{1} \times_{k} W_{2}}$ is the correspondence homomorphism from [VSF00, $\S$ II.3.7]. By [VSF00, Corollary II.3.7.5], we have

$$
c^{\prime} \cap \mathcal{Z}_{W_{2}} \in z_{\text {equi }}\left(W_{2}, W_{1} \times_{k} \mathbb{A}^{n}, 0\right)(U) .
$$




\section{THE BOUNDARY MOTIVE: DEFINITION AND BASIC PROPERTIES}

Furthermore, finiteness of $\mathcal{Z}$ over $U$ implies that $c^{\prime} \cap \mathcal{Z}_{W_{2}}$ is finite over $U \times_{k} W_{2} \times_{k} \mathbb{A}^{n}$. Push-forward via the projection $p_{2}$ to this product then yields

$$
e_{c^{\prime}}(\mathcal{Z}):=p_{2 *}\left(c^{\prime} \cap \mathcal{Z}_{W_{2}}\right) \in z_{\text {equi }}\left(W_{2}, \mathbb{A}^{n}, 0\right)(U) .
$$

In fact, $e_{c^{\prime}}(\mathcal{Z})$ lies in $z_{\text {equi }}\left(Y_{2}^{\bullet} \rightarrow W_{2}, \mathbb{A}^{n}, 0\right)(U)$ since $c^{\prime}$ has empty intersection with $W_{1} \times_{k} Y_{2}$. Furthermore, $e_{c^{\prime}}(\mathcal{Z})$ only depends on the class of $\mathcal{Z}$ modulo $c\left(U, Y_{1}\right)$ since $c^{\prime}$ has empty intersection with $Y_{1} \times_{k} W_{2}$.

(B) Let $W \in S m / k$ be of pure dimension, with a closed sub-scheme $Y \subset W$, such that arbitrary intersections of the components of $Y$ are smooth. Let

$$
c \in C y c^{n}\left(W \times_{k} W\right)_{Y \times_{k} W \cup W \times{ }_{k} Y} .
$$

Then a sufficient condition for the pairing

$$
(\bullet, \bullet)_{c}: M_{\mathrm{gm}}(W / Y) \otimes M_{\mathrm{gm}}(W / Y) \longrightarrow \mathbb{Z}(n)[2 n]
$$

to be symmetric or, equivalently, for the morphism

$$
\varepsilon_{c}: M_{\mathrm{gm}}(W / Y) \longrightarrow M_{\mathrm{gm}}(W / Y)^{*}(n)[2 n]
$$

to be auto-dual (i.e. $\left.\varepsilon_{c}=\varepsilon_{c}^{*}(n)[2 n]\right)$, is the symmetry of the cycle $c$ in $C y c^{n}\left(W \times_{k} W\right)$.

(C) For $i=1, \ldots, 4$, let $W_{i} \in S m / k$ be of pure dimension, with closed sub-schemes $Y_{i} \subset W_{i}$, such that arbitrary intersections of the components of the $Y_{i}$ are smooth. For $i=1,2$, let $j_{i}: W_{i} \hookrightarrow W_{i+2}$ be an open immersion mapping $Y_{i}$ into $Y_{i+2}$. Let

$$
c_{1,4} \in \operatorname{Cyc}^{n}\left(W_{1} \times_{k} W_{4}\right)_{Y_{1} \times_{k} W_{4} \cup W_{1} \times_{k} Y_{4}}
$$

and

$$
c_{3,2} \in C y c^{n}\left(W_{3} \times_{k} W_{2}\right)_{Y_{3} \times_{k} W_{2} \cup W_{3} \times{ }_{k} Y_{2}} .
$$

Then a sufficient condition for the diagram

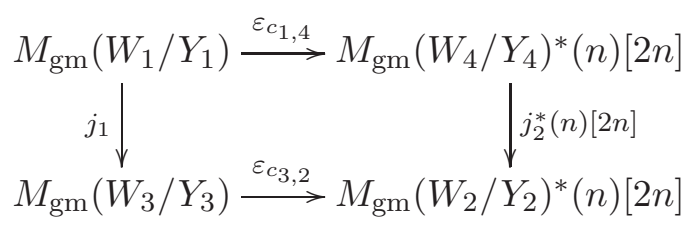

to commute is the equality of cycles

$$
\left(j_{1}, \operatorname{id}_{W_{2}}\right)^{*} c_{3,2}=\left(\operatorname{id}_{W_{1}}, j_{2}\right)^{*} c_{1,4}
$$

in $\operatorname{Cyc}^{n}\left(W_{1} \times_{k} W_{2}\right)$.

Furthermore, using the compatibility of moving cycles with correspondence homomorphisms and direct images, and [VSF00, Proposition II.3.7.6], one sees the following principle.

(D) For $i=1,2$, let $W_{i} \in S m / k$ be of pure dimension $m_{i}$, with a closed sub-scheme $Y_{1} \subset W_{1}$, such that arbitrary intersections of the components of $Y_{1}$ are smooth. Assume that $m_{2} \geqslant n$ and that

$$
c \in C y c^{n}\left(W_{1} \times_{k} W_{2}\right)_{Y_{1} \times_{k} W_{2}} \cap z_{\text {equi }}\left(W_{1}, W_{2}, m_{2}-n\right)(\operatorname{Spec} k) .
$$

Then the morphism

$$
\varepsilon_{c}: M_{\mathrm{gm}}\left(W_{1} / Y_{1}\right) \longrightarrow M_{\mathrm{gm}}\left(W_{2}\right)^{*}(n)[2 n]
$$

associated to $c$ is also induced by the composition of the morphism of Nisnevich sheaves

$$
e_{c}^{\prime}: c\left(\bullet, W_{1}\right) / c\left(\bullet, Y_{1}\right) \longrightarrow z_{\text {equi }}\left(W_{2} \times_{k} \mathbb{A}^{n}, m_{2}\right)
$$




\section{J. Wildeshaus}

with the inverse of the isomorphism $\mathbf{R} C(\mathcal{D})$ from Theorem 6.11 , where $e_{c}^{\prime}$ is defined as follows. Given $U \in S m / k$ and $\mathcal{Z} \in c\left(U, W_{1}\right)$, pull back $\mathcal{Z}$ to $\mathbb{A}^{n}$, giving $\mathcal{Z}_{\mathbb{A}^{n}} \in c\left(U \times{ }_{k} \mathbb{A}^{n}, W_{1}\right)$. Similarly, pull back $c$ to $U \times_{k} \mathbb{A}^{n}$, giving $c_{U \times_{k} \mathbb{A}^{n}}$. Now consider the cycle

$$
c \cap \mathcal{Z}_{\mathbb{A}^{n}}:=\operatorname{Cor}_{W_{1} \times_{k} W_{2} \times{ }_{k} \mathbb{A}^{n} / W_{1} \times{ }_{k} \mathbb{A}^{n}}\left(c_{U \times_{k} \mathbb{A}^{n}} \otimes \mathcal{Z}_{\mathbb{A}^{n}}\right) .
$$

By [VSF00, Corollary II.3.7.5], we have

$$
c \cap \mathcal{Z}_{\mathbb{A}^{n}} \in z_{\text {equi }}\left(\mathbb{A}^{n}, W_{1} \times_{k} W_{2}, m_{2}-n\right)(U) .
$$

Furthermore, finiteness of $\mathcal{Z}$ over $U$ implies that $c \cap \mathcal{Z}_{\mathbb{A}^{n}}$ is finite over $U \times_{k} W_{2} \times_{k} \mathbb{A}^{n}$. Push-forward via the projection $p_{2}$ to this product then yields

$$
e_{c}^{\prime}(\mathcal{Z}):=p_{2 *}\left(c \cap \mathcal{Z}_{\mathbb{A}^{n}}\right) \in z_{\text {equi }}\left(\mathbb{A}^{n}, W_{2}, m_{2}-n\right)(U),
$$

and the latter group is contained in $z_{\text {equi }}\left(W_{2} \times{ }_{k} \mathbb{A}^{n}, m_{2}\right)(U)$. Observe that $e_{c}^{\prime}(\mathcal{Z})$ only depends on the class of $\mathcal{Z}$ modulo $c\left(U, Y_{1}\right)$ since $c$ has empty intersection with $Y_{1} \times_{k} W_{2}$.

Choose and fix a smooth compactification $\bar{X}$ of $X$ such that $\partial \bar{X}:=\bar{X}-X$ is a normal crossing divisor with smooth irreducible components. By [VSF00, Proposition V.4.1.5], we have a canonical isomorphism between $M_{\mathrm{gm}}(\bar{X} / \partial \bar{X})$ and $M_{\mathrm{gm}}^{\mathrm{c}}(X)$. Applying principle (A), we see that the diagonal

$$
\Delta \in \operatorname{Cyc}^{n}\left(\bar{X} \times_{k} X\right)_{\partial \bar{X} \times_{k} X}
$$

induces a morphism

$$
\varepsilon_{\Delta}: M_{\mathrm{gm}}^{\mathrm{c}}(X) \longrightarrow M_{\mathrm{gm}}(X)^{*}(n)[2 n] .
$$

Principle (D) and the proof of [VSF00, Theorem V.4.3.7] show that this is the morphism $\mu_{X}$. To say that

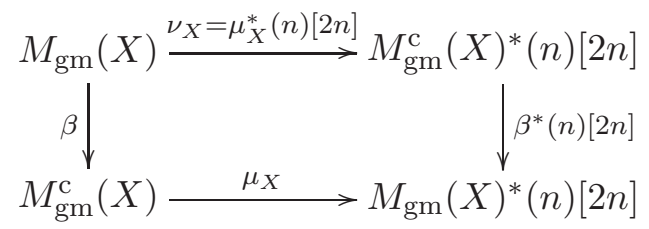

commutes is equivalent to saying that the pairing

$$
M_{\mathrm{gm}}(X) \otimes M_{\mathrm{gm}}(X) \stackrel{\mathrm{id} \otimes \beta}{\longrightarrow} M_{\mathrm{gm}}(X) \otimes M_{\mathrm{gm}}^{\mathrm{c}}(X) \stackrel{(\bullet, \bullet)_{\Delta}}{\longrightarrow} \mathbb{Z}(n)[2 n]
$$

is symmetric. By principle (B), this is indeed the case since the restriction of the cycle $\Delta$ to $X \times_{k} X$ is symmetric.

Now for the construction of

$$
\eta_{X}: \partial M_{\mathrm{gm}}(X) \longrightarrow \partial M_{\mathrm{gm}}(X)^{*}(n)[2 n-1] .
$$

Recall that by Proposition 2.4, there is a canonical isomorphism

$$
\text { Cone }\left(M_{\mathrm{gm}}(X \coprod \partial \bar{X}) \rightarrow M_{\mathrm{gm}}(\bar{X})\right) \stackrel{\sim}{\longrightarrow} \partial M_{\mathrm{gm}}(X) .
$$

From this, one deduces that $\partial M_{\mathrm{gm}}(X) \otimes \partial M_{\mathrm{gm}}(X)[1]$ is represented by the complex $\underline{C}_{*}\left(s \mathcal{L}_{*}\right)$, where $\mathcal{L}_{*}$ is the complex concentrated in degrees $-1,0$ and 1 :

$$
\begin{gathered}
L\left(X \times_{k} X\right) \oplus L\left(X \times_{k} \partial \bar{X}\right) \oplus L\left(\partial \bar{X} \times_{k} X\right) \oplus L\left(\partial \bar{X} \times_{k} \partial \bar{X}\right) \\
L\left(\bar{X} \times_{k} X\right) \oplus L\left(X \times_{k} \bar{X}\right) \oplus L\left(\bar{X} \times_{k} \partial \bar{X}\right) \oplus L\left(\partial \bar{X} \times_{k} \bar{X}\right) \\
\left.\downarrow_{L}^{\downarrow} \times_{k} \bar{X}\right)
\end{gathered}
$$




\section{THE BOUNDARY MOTIVE: DEFINITION AND BASIC PROPERTIES}

with the differentials being induced by the inclusions. In particular, one sees that the complex $\mathcal{L}_{*}^{\prime}$ concentrated in degrees -1 and 0 , that is

$$
\begin{gathered}
L\left(X \times_{k} X\right) \oplus L\left(X \times_{k} \partial \bar{X}\right) \oplus L\left(\partial \bar{X} \times_{k} X\right) \\
\downarrow \\
L\left(\bar{X} \times_{k} X\right) \oplus L\left(X \times_{k} \bar{X}\right),
\end{gathered}
$$

is a quotient of $\mathcal{L}_{*}$. By the Mayer-Vietoris property for the functor $M_{\mathrm{gm}}$ [VSF00, Proposition V.4.1.1], $\underline{C}_{*}\left(\mathcal{L}_{*}^{\prime}\right)$ is canonically quasi-isomorphic to the complex $\underline{C}_{*}\left(\mathcal{L}_{*}^{\prime \prime}\right)$, where $\mathcal{L}_{*}^{\prime \prime}$ is the complex concentrated in degrees -1 and 0 :

$$
\begin{gathered}
L\left(\bar{X} \times_{k} \bar{X}-\partial \bar{X} \times_{k} \partial \bar{X}-X \times_{k} X\right) \\
\downarrow \\
L\left(\bar{X} \times_{k} \bar{X}-\partial \bar{X} \times_{k} \partial \bar{X}\right) .
\end{gathered}
$$

This shows that there is a canonical morphism from $\partial M_{\mathrm{gm}}(X) \otimes \partial M_{\mathrm{gm}}(X)[1]$ to

$$
M_{\mathrm{gm}}\left(\left(\bar{X} \times_{k} \bar{X}-\partial \bar{X} \times_{k} \partial \bar{X}\right) /\left(\bar{X} \times_{k} \bar{X}-\partial \bar{X} \times_{k} \partial \bar{X}-X \times_{k} X\right)\right) .
$$

Applying principle (A), we see that the diagonal

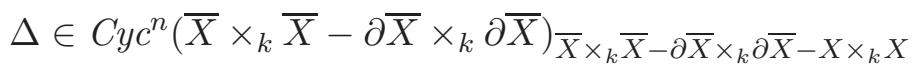

induces a morphism

$$
\varepsilon_{\Delta}: \partial M_{\mathrm{gm}}(X) \longrightarrow \partial M_{\mathrm{gm}}(X)^{*}(n)[2 n-1] .
$$

We define this to be the morphism $\eta_{X}$. Principle (B) shows that $\eta_{X}$ is auto-dual. In order to see that $\eta_{X}$ fits into a morphism of exact triangles

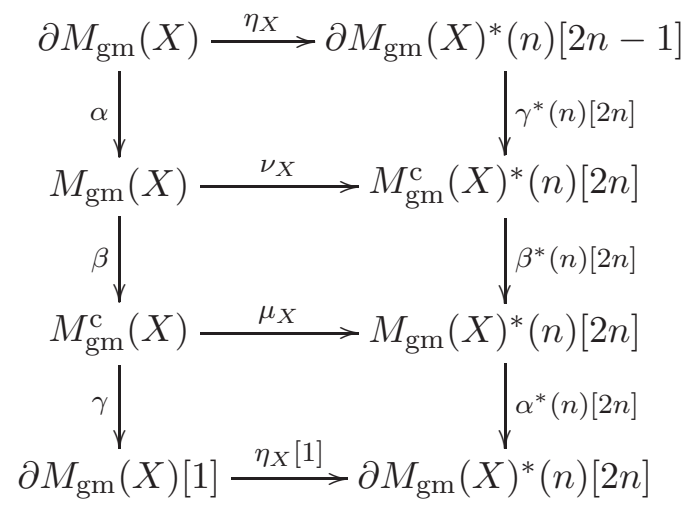

apply principle (C). By [VSF00, Theorem V.4.3.7 3], $\mu_{X}$ and $\nu_{X}$ are isomorphisms, and hence so is $\eta_{X}$. In order to check that $\eta_{X}$ does not depend on the choice of the smooth compactification $\bar{X}$, use Remark 6.15 together with the fact that the system of such compactifications is filtering.

Fix a compactification $\bar{X}$ of $X$ (which may be non-smooth) and set $\partial \bar{X}:=\bar{X}-X$. Denote by $j$ the open immersion of $X$ and by $i_{\partial \bar{X}}$ for the closed immersion of $\partial \bar{X}$ into $\bar{X}^{m}$. We are thus in the situation considered in $\S 3$, with $Y=Y^{\prime}=\partial \bar{X}$ and $W=\bar{X}$. By Proposition 2.4, we have a canonical isomorphism

By duality, this gives

$$
M_{\mathrm{gm}}\left(\partial \bar{X}, i_{\partial \bar{X}}^{!} j_{!} \mathbb{Z}\right) \stackrel{\sim}{\longrightarrow} \partial M_{\mathrm{gm}}(X)[1]
$$

$$
\alpha_{X}: \partial M_{\mathrm{gm}}(X)^{*}[-1] \stackrel{\sim}{\longrightarrow} M_{\mathrm{gm}}\left(\partial \bar{X}, i_{\partial \bar{X}}^{!} j ! \mathbb{Z}^{*}\right.
$$




\section{J. Wildeshaus}

Preceding $\alpha_{X}(n)[2 n]$ with the auto-duality $\eta_{X}$ from Theorem 6.1, we obtain the following.

COROllary 6.16. There is a canonical isomorphism

$$
\partial M_{\mathrm{gm}}(X) \stackrel{\sim}{\longrightarrow} M_{\mathrm{gm}}\left(\partial \bar{X}, i_{\partial \bar{X}}^{!} j_{!} \mathbb{Z}\right)^{*}(n)[2 n] .
$$

\section{Localization}

Throughout this section, we assume that $k$ admits resolution of singularities. Fix closed immersions $Y \hookrightarrow Y^{\prime} \hookrightarrow W$ in $S c h / k$. Denote by $j$ the open immersion of $W-Y^{\prime}$ and by $i_{Y}$ the closed immersion of $Y$ into $W$.

Definition 7.1. Assume that $W-Y^{\prime} \in S m / k$.

(a) If $W-Y^{\prime}$ is of pure dimension $n$, we put

$$
M_{\mathrm{gm}}^{\mathrm{c}}\left(Y, i_{Y}^{*} j_{*} \mathbb{Z}\right):=M_{\mathrm{gm}}\left(Y, i_{Y}^{!} j ! \mathbb{Z}\right)^{*}(n)[2 n] .
$$

(b) If $W-Y^{\prime}=\coprod_{\alpha} W_{\alpha}$ is the decomposition of $W-Y^{\prime}$ into connected components, then the motive with compact support of $Y$ and with coefficients in $i_{Y}^{*} j_{*} \mathbb{Z}$ is defined as

$$
M_{\mathrm{gm}}^{\mathrm{c}}\left(Y, i_{Y}^{*} j_{*} \mathbb{Z}\right):=\bigoplus_{\alpha} M_{\mathrm{gm}}^{\mathrm{c}}\left(Y, i_{Y}^{*} j_{\alpha *} \mathbb{Z}\right) .
$$

Here, the $j_{\alpha}$ denote the open immersions of the $W_{\alpha}$ into $W$.

Using [VSF00, Corollary V.4.3.6] and the definition of $M_{\mathrm{gm}}\left(Y, i_{Y}^{!} j_{!} \mathbb{Z}\right)$ (Definition 3.1), one sees that $M_{\mathrm{gm}}^{\mathrm{c}}\left(Y, i_{Y}^{*} j_{*} \mathbb{Z}\right)$ belongs to $D M_{\mathrm{gm}}^{\mathrm{eff}}(k)$.

Remark 7.2. The object on the right-hand side in Definition 7.1 is defined without the hypothesis of smoothness on $W-Y^{\prime}$. In general, the object $M_{\mathrm{gm}}\left(Y, i_{Y}^{!} j_{!} \mathbb{Z}\right)$ is dual to what should be considered as the motive with compact support of $Y$ and with coefficients in $i_{Y}^{*} j_{*} \mathbb{D}(\mathbb{Z})$, where $\mathbb{D}(\mathbb{Z})$ is the coefficient system on $W-Y^{\prime}$ which is Verdier-dual to $\mathbb{Z}$.

Now assume a given filtration

$$
\emptyset=\mathfrak{F}_{-1} Y \subset \mathfrak{F}_{0} Y \subset \cdots \subset \mathfrak{F}_{d} Y=Y
$$

of $Y$ by closed sub-schemes. It induces a stratification of $Y$ by locally closed sub-schemes $Y_{\mathrm{m}}:=$ $\mathfrak{F}_{\mathrm{m}} Y-\mathfrak{F}_{\mathrm{m}-1} Y$, for $m=0, \ldots, d$. Define $W^{m}$ as the complement of $\mathfrak{F}_{\mathrm{m}-1} Y$ in $W$. Write $i_{Y_{\mathrm{m}}}$ for the closed immersion of $Y_{\mathrm{m}}$ into $W^{m}$. By abuse of notation, we use the letter $j$ to also denote the open immersions of $W-Y^{\prime}$ into $W^{m}$.

Theorem 7.3 (Localization). Assume that $W-Y^{\prime} \in S m / k$. Then there is a canonical chain of morphisms

$$
M_{0}=M_{\mathrm{gm}}^{\mathrm{c}}\left(Y, i_{Y}^{*} j_{*} \mathbb{Z}\right) \stackrel{\gamma_{0}}{\longrightarrow} M_{1} \stackrel{\gamma_{1}}{\longrightarrow} M_{2} \stackrel{\gamma_{2}}{\longrightarrow} \cdots \stackrel{\gamma_{d-1}}{\longrightarrow} M_{d} \stackrel{\gamma_{d}}{\longrightarrow} M_{d+1}=0
$$

in $D M_{\mathrm{gm}}(k)$. For each $m \in\{0, \ldots, d\}$, there is a canonical exact triangle

$$
M_{\mathrm{gm}}^{\mathrm{c}}\left(Y_{\mathrm{m}}, i_{Y_{\mathrm{m}}}^{*} j_{*} \mathbb{Z}\right) \longrightarrow M_{\mathrm{m}} \stackrel{\gamma_{\mathrm{m}}}{\longrightarrow} M_{\mathrm{m}+1} \longrightarrow M_{\mathrm{gm}}^{\mathrm{c}}\left(Y_{\mathrm{m}}, i_{Y_{\mathrm{m}}}^{*} j_{*} \mathbb{Z}\right)[1]
$$

in $D M_{\mathrm{gm}}(k)$. In particular, all the $M_{\mathrm{m}}$ are in $D M_{\mathrm{gm}}^{\mathrm{eff}}(k)$.

Proof. Dualize co-localization (Theorem 3.4).

Corollary 7.4. In the above situation, assume that $Y=\partial \bar{X}:=\bar{X}-X$, with $X \in S m / k$, and $\bar{X}$ a compactification of $X$ (which may be non-smooth). Write $(\partial \bar{X})_{\mathrm{m}}:=Y_{\mathrm{m}}$. Then there is a canonical chain of morphisms

$$
M_{0}=\partial M_{\mathrm{gm}}(X) \stackrel{\gamma_{0}}{\longrightarrow} M_{1} \stackrel{\gamma_{1}}{\longrightarrow} M_{2} \stackrel{\gamma_{2}}{\longrightarrow} \cdots \stackrel{\gamma_{d-1}}{\longrightarrow} M_{d} \stackrel{\gamma_{d}}{\longrightarrow} M_{d+1}=0
$$




\section{THE BOUNDARY MOTIVE: DEFINITION AND BASIC PROPERTIES}

in $D M_{\mathrm{gm}}(k)$. For each $m \in\{0, \ldots, d\}$, there is a canonical exact triangle

$$
M_{\mathrm{gm}}^{\mathrm{c}}\left((\partial \bar{X})_{\mathrm{m}}, i_{(\partial \bar{X})_{\mathrm{m}}} j_{*} \mathbb{Z}\right) \longrightarrow M_{\mathrm{m}} \stackrel{\gamma_{\mathrm{m}}}{\longrightarrow} M_{\mathrm{m}+1} \longrightarrow M_{\mathrm{gm}}^{\mathrm{c}}\left((\partial \bar{X})_{\mathrm{m}}, i_{(\partial \bar{X})_{\mathrm{m}}}^{*} j_{*} \mathbb{Z}\right)[1]
$$

in $D M_{\mathrm{gm}}(k)$. In particular, all the $M_{\mathrm{m}}$ are in $D M_{\mathrm{gm}}^{\mathrm{eff}}(k)$.

Proof. This follows from Corollary 6.16 and Theorem 7.3.

Remark 7.5. Given our definition, it is easy to deduce from duality and Theorems 4.1 and 5.1 that $M_{\mathrm{gm}}^{\mathrm{c}}\left(Y, i_{Y}^{*} j_{*} \mathbb{Z}\right)$ is invariant under abstract blow-up and under analytical isomorphism. In concrete situations, this observation helps to control the 'graded pieces' $M_{\mathrm{gm}}^{\mathrm{c}}\left((\partial \bar{X})_{\mathrm{m}}, i_{(\partial \bar{X})_{\mathrm{m}}} j_{*} \mathbb{Z}\right)$ of $\partial M_{\mathrm{gm}}(X)$.

\section{The case of normal crossings}

Throughout this section, we consider the following situation: $X$ lies in $S m / k$ and is irreducible and $\bar{X} \in S m / k$ is a smooth compactification of $X$, such that $\partial \bar{X}:=\bar{X}-X$ is a normal crossing divisor with smooth irreducible components. We stratify $\partial \bar{X}$ by defining $(\partial \bar{X})^{m}$ as the geometric locus of points lying on exactly $m$ irreducible components, $m=1, \ldots, \operatorname{dim} X$. Note that the $(\partial \bar{X})^{m}$ are all smooth. Denote by $j$ the open immersion of $X$, and by $i_{\mathrm{m}}$ the immersion of $(\partial \bar{X})^{m}$ into $\bar{X}$. We re-state co-localization and localization (Corollaries 3.5 and 7.4) as follows.

Corollary 8.1. Denote by $n$ the dimension of $X$, and assume that $k$ admits resolution of singularities.

(a) There is a canonical chain of morphisms

$$
M^{n}=0 \stackrel{\gamma^{n-1}}{\longrightarrow} M^{n-1} \stackrel{\gamma^{n-2}}{\longrightarrow} M^{n-2} \stackrel{\gamma^{n-3}}{\longrightarrow} \cdots \stackrel{\gamma^{0}}{\longrightarrow} M^{0}=\partial M_{\mathrm{gm}}(X)[1]
$$

in $D M_{\mathrm{gm}}^{\mathrm{eff}}(k)$. For each $m \in\{1, \ldots, n\}$, there is a canonical exact triangle

$$
M_{\mathrm{gm}}\left((\partial \bar{X})^{m}, i_{\mathrm{m}}^{!} j_{!} \mathbb{Z}\right)[-1] \longrightarrow M^{n-m+1} \stackrel{\gamma^{n-m}}{\longrightarrow} M^{n-m} \longrightarrow M_{\mathrm{gm}}\left((\partial \bar{X})^{m}, i_{\mathrm{m}}^{!} j_{!} \mathbb{Z}\right)
$$

in $D M_{\mathrm{gm}}^{\mathrm{eff}}(k)$.

(b) There is a canonical chain of morphisms

$$
M_{0}=\partial M_{\mathrm{gm}}(X) \stackrel{\gamma_{0}}{\longrightarrow} M_{1} \stackrel{\gamma_{1}}{\longrightarrow} M_{2} \stackrel{\gamma_{2}}{\longrightarrow} \cdots \stackrel{\gamma_{n-2}}{\longrightarrow} M_{n-1} \stackrel{\gamma_{n-1}}{\longrightarrow} M_{n}=0
$$

in $D M_{\mathrm{gm}}^{\mathrm{eff}}(k)$. For each $m \in\{1, \ldots, n\}$, there is a canonical exact triangle

$$
M_{\mathrm{gm}}^{\mathrm{c}}\left((\partial \bar{X})^{m}, i_{\mathrm{m}}^{*} j_{*} \mathbb{Z}\right) \longrightarrow M_{n-m} \stackrel{\gamma_{n-m}}{\longrightarrow} M_{n-m+1} \longrightarrow M_{\mathrm{gm}}^{\mathrm{c}}\left((\partial \bar{X})^{m}, i_{\mathrm{m}}^{*} j_{*} \mathbb{Z}\right)[1]
$$

in $D M_{\mathrm{gm}}^{\mathrm{eff}}(k)$.

The aim of this section is to give a description of the motives with coefficients $M_{\mathrm{gm}}\left((\partial \bar{X})^{m}\right.$, $\left.i_{\mathrm{m}}^{!} j_{!} \mathbb{Z}\right)$ and $M_{\mathrm{gm}}^{\mathrm{c}}\left((\partial \bar{X})^{m}, i_{\mathrm{m}}^{*} j_{*} \mathbb{Z}\right)$ occurring in the above statement. Denote by $N_{\mathrm{m}}$ the normal bundle of $(\partial \bar{X})^{m}$ in $\bar{X}$. For any component $D$ of the divisor $\partial \bar{X}$, consider the normal bundle of $(\partial \bar{X})^{m} \cap D$ in $D$. Using all possible $D$, this gives $m$ sub-bundles of $N_{\mathrm{m}}$ of codimension one. Their intersection is the zero bundle over $(\partial \bar{X})^{m}$. Define $N_{\mathrm{m}}^{*}$ as the complement in $N_{\mathrm{m}}$ of the union of these sub-bundles. Fix an order $\prec$ of the index set of components of $\partial \bar{X}$, i.e. write

$$
\partial \bar{X}=\bigcup_{i=1}^{r} D_{i},
$$

where $D_{1}, \ldots, D_{r}$ are the $r$ distinct components of $\partial \bar{X}$. Note that $N_{\mathrm{m}}^{*}$ is a torsor under a torus of dimension $m$, and that the choice of $\prec$ gives an identification of this torus with $\mathbb{G}_{\mathrm{m}}^{m}$. 


\section{J. Wildeshaus}

\section{THEOREM 8.2.}

(a) The order $\prec$ induces an isomorphism

$$
M_{\mathrm{gm}}\left((\partial \bar{X})^{m}, i_{\mathrm{m}}^{!} j_{!} \mathbb{Z}\right) \stackrel{\sim}{\longrightarrow} M_{\mathrm{gm}}\left(N_{\mathrm{m}}^{*}\right)[m]
$$

in $D M_{\mathrm{gm}}^{\mathrm{eff}}(k)$.

(a) Assume that $k$ admits resolution of singularities. Then the order $\prec$ induces an isomorphism

$$
M_{\mathrm{gm}}^{\mathrm{c}}\left((\partial \bar{X})^{m}, i_{\mathrm{m}}^{*} j_{*} \mathbb{Z}\right) \stackrel{\sim}{\longrightarrow} M_{\mathrm{gm}}^{\mathrm{c}}\left(N_{\mathrm{m}}^{*}\right)[-m]
$$

in $D M_{\mathrm{gm}}^{\mathrm{eff}}(k)$.

Our main computational tool in the proof of Theorem 8.2 will be the following.

Lemma 8.3. Let $S \in S c h / k$ and let $V$ be a vector bundle of rank $m$ over $S$. Assume given subbundles $V_{i}, i=1, \ldots, \ell$ of codimension one of $V$, with $1 \leqslant \ell \leqslant m$. Assume also that Zariski-locally over $S$, there exist trivializations $V \cong \mathbb{A}_{S}^{m}$ identifying $V_{i}$ with the hyperplane given by the vanishing of the $i$ th coordinate, $i=1, \ldots, \ell$. Define $V^{*}$ as the complement in $V$ of the union of the $V_{i}$. Then for any proper subset $I$ of $\{1, \ldots, \ell\}$, the immersion $V-V^{*}-\cup_{i \in I} V_{i} \hookrightarrow V-\cup_{i \in I} V_{i}$ induces an isomorphism

$$
M_{\mathrm{gm}}\left(V-V^{*}-\cup_{i \in I} V_{i}\right) \stackrel{\sim}{\longrightarrow} M_{\mathrm{gm}}\left(V-\cup_{i \in I} V_{i}\right)
$$

in $D M_{\mathrm{gm}}^{\mathrm{eff}}(k)$.

Proof. The Mayer-Vietoris property [VSF00, Proposition V.3.1.3] for the functor $L$ shows that we may assume that a trivialization as in the hypotheses exists globally. Then the immersion $V^{*} \hookrightarrow V-\bigcup_{i \in I} V_{i}$ is isomorphic to

$$
\mathbb{G}_{m, S}^{\ell} \times \mathbb{A}_{S}^{m-\ell} \longleftrightarrow \mathbb{G}_{m, S}^{p} \times{ }_{S} \mathbb{A}_{S}^{m-p}
$$

for some $p<\ell$. Hence $V-V^{*}-\cup_{i \in I} V_{i} \hookrightarrow V-\cup_{i \in I} V_{i}$ is a homotopy equivalence (in fact, both sides are homotopic to $\left.\mathbb{G}_{m, S}^{p}\right)$.

Proof of Theorem 8.2. (a) Recall that $M_{\mathrm{gm}}\left((\partial \bar{X})^{m}, i_{\mathrm{m}}^{!} j_{!} \mathbb{Z}\right)$ is defined as the motive $M_{\mathrm{gm}}(\mathfrak{Y})$, where $\mathfrak{Y}$ is the diagram

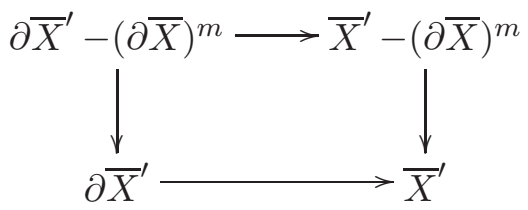

and $\bar{X}^{\prime}$ and $\partial \bar{X}^{\prime}$ denote the complements of the strata $(\partial \bar{X})^{p}$, for $p>m$. Now because of our assumptions on $\bar{X}$ and $\partial \bar{X}$, this diagram is analytically isomorphic to the following diagram $\mathfrak{N}$, in the sense that the assumptions of Theorem 5.1 are satisfied.

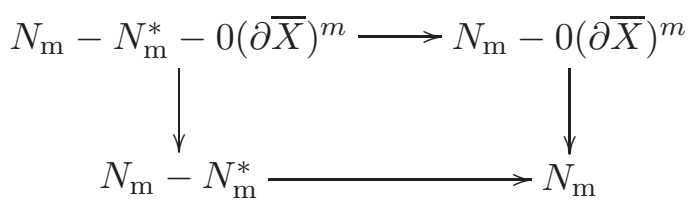

Here, $0(\partial \bar{X})^{m}$ denotes the zero section of the bundle $N_{\mathrm{m}}$ over $(\partial \bar{X})^{m}$. Analytical invariance thus allows us to compute $M_{\mathrm{gm}}(\mathfrak{N})$ instead of $M_{\mathrm{gm}}(\mathfrak{Y})$. In order to do so, consider the upper line

$$
N_{\mathrm{m}}-N_{\mathrm{m}}^{*}-0(\partial \bar{X})^{m} \longrightarrow N_{\mathrm{m}}-0(\partial \bar{X})^{m}
$$


The BOUNDARY MOTIVE: DEFINITION AND BASIC PROPERTIES

of $\mathfrak{N}$, and the finite covering

$$
N_{\mathrm{m}}-0(\partial \bar{X})^{m}=\bigcup_{i=1}^{m} N_{\mathrm{m}}-V_{i} .
$$

Here, the $V_{i}$ are the sub-bundles of codimension one constructed from the components of $\partial \bar{X}$. For $1 \leqslant i_{1}<\cdots<i_{p} \leqslant m$, we have

$$
\bigcap_{q=1}^{p} N_{\mathrm{m}}-V_{i_{q}}=N_{\mathrm{m}}-\bigcup_{q=1}^{p} V_{i_{q}} .
$$

Define the double complex $L\left(\mathfrak{N}^{\prime}\right)$ of Nisnevich sheaves as follows. First consider the Cech complex associated to the above covering, and to the induced covering of $N_{\mathrm{m}}-N_{\mathrm{m}}^{*}-0(\partial \bar{X})^{m}$, then add the lower line of $L(\mathfrak{N})$ as follows.

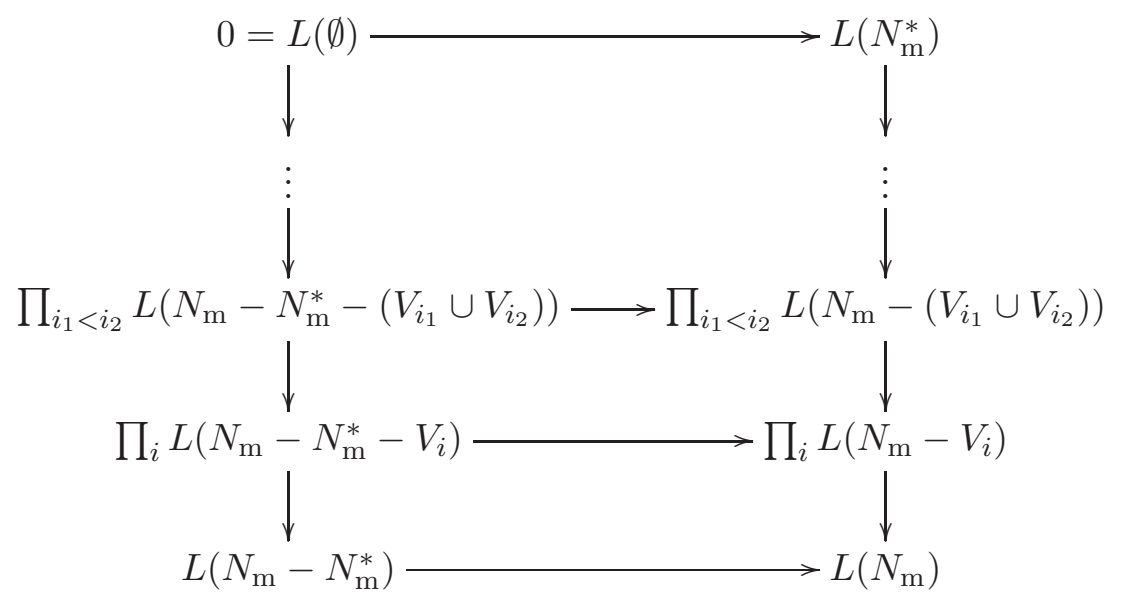

Here, we have total degree zero for $L\left(N_{\mathrm{m}}\right)$, hence degree $-m$ for $L\left(N_{\mathrm{m}}^{*}\right)$. We thus get a canonical morphism of complexes of Nisnevich sheaves

$$
L\left(\mathfrak{N}^{\prime}\right) \longrightarrow L(\mathfrak{N})
$$

The Mayer-Vietoris property [VSF00, Proposition V.3.1.3] shows that it induces an isomorphism in $D M_{-}^{\text {eff }}(k)$. By Lemma 8.3, the projection from $L\left(\mathfrak{N}^{\prime}\right)$ to its upper line

also induces an isomorphism in $D M_{-}^{\text {eff }}(k)$

$$
L\left(\mathfrak{N}^{\prime}\right) \longrightarrow L\left(N_{\mathrm{m}}^{*}\right)[m]
$$

(b) results from (a) by dualizing.

As the proof of Theorem 8.2 shows, the dependence of the isomorphism on the order $\prec$ can be made explicit. A canonical isomorphism between $M_{\mathrm{gm}}\left((\partial \bar{X})^{m}, i_{\mathrm{m}}^{!} j_{!} \mathbb{Z}\right)$ and a twisted form of $M_{\mathrm{gm}}\left(N_{\mathrm{m}}^{*}\right)[m]$ can be constructed by proceeding as in [Del71, (3.1.4)]. We leave the details to the reader.

\section{ACKNowledGements}

This work was done while I was enjoying a délégation auprès $d u C N R S$, and during visits to the Sonderforschungsbereich 478 of the University of Münster, and to the Institut de Matemàtica of the University of Barcelona. I am grateful to all three institutions. I also wish to thank J. Barge, J. I. Burgos, C. Deninger, F. Lemma, M. Levine and F. Morel for useful discussions and comments. The remarks of the referee were appreciated; it is thanks to her or him that $\S 8$ (on the case of a normal crossing boundary $\partial \bar{X}$ ) was added. 


\section{REFERENCES}

Art69 M. Artin, Algebraic approximation of structures over complete local rings, Publ. Math. Inst. Hautes Études Sci. 36 (1969), 23-58.

Del71 P. Deligne, Théorie de Hodge, II, Publ. Math. Inst. Hautes Études Sci. 40 (1971), 5-57.

Har93 G. Harder, Eisenstein Kohomologie und die Konstruktion gemischter Motive, Lecture Notes in Mathematics, vol. 1562 (Springer, Berlin, 1993).

Ver85 J.-L. Verdier, Extension of a perverse sheaf over a closed subspace, Astérisque 130 (1985), 210-217.

VSF00 V. Voevodsky, A. Suslin and E. M. Friedlander, Cycles, transfers, and motivic homology theories, Annals of Mathematics Studies, vol. 143 (Princeton University Press, Princeton, NJ, 2000).

Wil04 J. Wildeshaus, On the boundary motive of a Shimura variety, Preprint (2004), arXiv:math.AG/0409400.

Jörg Wildeshaus wildesh@math.univ-paris13.fr

Institut Galilée, Université Paris 13, Avenue Jean-Baptiste Clément, F-93430 Villetaneuse, France 